\title{
ON THE COMPOSITE DISCONTINUOUS GALERKIN METHOD FOR SIMULATIONS OF ELECTRIC PROPERTIES OF SEMICONDUCTOR DEVICES*
}

\author{
KONRAD SAKOWSKI ${ }^{\dagger \ddagger}$, LESZEK MARCINKOWSKI ${ }^{\ddagger}$, PAWEL STRAK ${ }^{\dagger}$, PAWEL KEMPISTY $^{\dagger}$, AND
}

STANISLAW KRUKOWSKI ${ }^{\dagger}$

\begin{abstract}
In this paper, a variant of the discretization of the van Roosbroeck equations in the equilibrium state with the composite discontinuous Galerkin method for the rectangular domain is discussed. It is based on the symmetric interior penalty Galerkin (SIPG) method. The proposed method accounts for lower regularity of the solution at the interfaces of the layers of the device. It is shown that the discrete problem is well defined and that the discrete solution is unique. Error estimates are derived. Finally, numerical simulations are presented.
\end{abstract}

Key words. composite discontinuous Galerkin method, drift-diffusion, van Roosbroeck equations

AMS subject classifications. 65N30, 65N15, 82D37

1. Introduction. Numerical simulations are an important tool in the development of semiconductor devices. Since our contemporary electronics rely on semiconductors, there is a strong demand for progress in this domain. Examples of such devices are light emitting diodes, lasers, transistors, detectors, and many others. There are various approaches for the simulation of such devices depending on precision, efficiency, and the size of the simulated fragment. On the one hand, there are so-called ab initio methods, which are used to investigate properties of elements composed of hundreds of thousands of atoms. These methods use fundamental laws of physics, and they need days or weeks to perform a single simulation on a computational cluster. Then there is drift-diffusion theory. In this case, the model is much simpler, and it allows for simulating whole semiconductor devices on a standard desktop computer. This model describes two kinds of carriers (electrons and holes), which move in the electric field present in semiconductor devices. From the mathematical point of view, it consists of a system of three nonlinear elliptic differential equations, which are called the van Roosbroeck equations [29].

Numerical modeling of semiconductor devices with the drift-diffusion model has been performed since 1964, when Gummel [11] proposed a numerical algorithm based on a simple iteration method. Various methods were used for the discretization of the van Roosbroeck equations, for example, the finite difference method (FDM) [26], the box method [1], or the finite element method (FEM) [4]. Special variants of discretizations optimized for the so-called continuity equations were developed [19].

In this paper, we focus our analysis on the following nonlinear elliptic equation for $u^{*}$,

$$
-\nabla \cdot\left(\varepsilon \nabla u^{*}\right)+e^{u^{*}-v^{*}}-e^{w^{*}-u^{*}}=k_{1},
$$

which is a special case of the van Roosbroeck problem: Find $u^{*}, v^{*}, w^{*}$ such that

$$
\begin{aligned}
-\nabla \cdot\left(\varepsilon \nabla u^{*}\right)+e^{u^{*}-v^{*}}-e^{w^{*}-u^{*}} & =k_{1}, \\
-\nabla \cdot\left(\mu_{n} e^{u^{*}-v^{*}} \nabla v^{*}\right)-Q\left(u^{*}, v^{*}, w^{*}\right)\left(e^{w^{*}-v^{*}}-1\right) & =0, \\
-\nabla \cdot\left(\mu_{p} e^{w^{*}-u^{*}} \nabla w^{*}\right)+Q\left(u^{*}, v^{*}, w^{*}\right)\left(e^{w^{*}-v^{*}}-1\right) & =0 .
\end{aligned}
$$

\footnotetext{
*Received on July 26, 2017. Accepted February 4, 2019. Published online on March 19, 2019. Recommended by S. Brenner.

${ }^{\dagger}$ Institute of High Pressure Physics, Polish Academy of Sciences, ul. Sokolowska 29/37, 01-142 Warsaw, Poland

${ }^{\ddagger}$ Faculty of Mathematics, Informatics and Mechanics, University of Warsaw, ul. Banacha 2, 02-097 Warsaw, Poland

${ }^{\S}$ Research Institute for Applied Mechanics, Kyushu University, Kasuga, Fukuoka 816-8580, Japan konrad.sakowski@unipress.waw.pl.
} 
The functions $\varepsilon(x), \mu_{n}(x), \mu_{p}(x), k_{1}(x)$ are material parameters, and $Q(x, u, v, w)$ is an operator depending on the semiconductor material. We do not want to discuss properties of these equations; we refer to $[18,26]$ for the physical details and to [13] for the mathematical background. In the equilibrium case, when there is no energy exchange between the simulated device and the environment, the functions $v^{*}, w^{*}$, which correspond to the quasi-Fermi levels $[18,21]$, are constant due to the physical nature of this problem and the system (1.2) simplifies to (1.1).

We would like to emphasize the main problems with the numerical solution of (1.2). The first issue is the nonlinearity. Depending on the device composition and design, the coefficients of the latter two equations may vary by several orders of magnitude. There are various approaches to the solution of this system. They may involve decoupling, Banach iteration [11, 20], Newton's method [14], etc. In this paper, we do not want to go into detail about this problem. For the specific solution method used by us in the numerical simulations, please refer to [23].

The problem we discuss here is the discretization of these equations. As mentioned, FDM and FEM discretizations are successfully used for this system since the second half of the 20 th century $[21,26]$. However, the design of semiconductor devices has been substantially changed over time. Initially, semiconductor transistors or diodes were made from a single material (e.g., silicon) divided into layers with different doping levels. These conditions were mathematically reflected by the $k_{1}$ function, possibly discontinuous, while $\varepsilon, \mu_{n}, \mu_{p}$ remained constant. On the other hand, contemporary semiconductor devices, like blue laser diodes (see Figure 2.1), consist of layers of different semiconductor materials deposited on top of each other. Recent designs also involve a change of material within one layer. The material parameters, like $\varepsilon, \mu_{n}, \mu_{p}$, are no longer constant. In general, they are discontinuous. However, these discontinuities are localized at the interfaces of the layers, and inside a layer, these parameters are constant or, in general, smooth functions.

Thus, to obtain a good precision, it would be advantageous to use a discretization which takes into account such localized lack of regularity and the discontinuities of the coefficients on the interfaces and which allows the exploitation of higher regularity inside the layers. A natural discretization method for such a problem would be the discontinuous Galerkin method (DGM) [5, 22]. However, this method by its nature imposes many more degrees of freedom in the simulations, leading to slower and more memory-consuming computations. Since the physical layers of semiconductor devices have regular shapes, it is feasible to use the composite discontinuous Galerkin method (CDGM) [6], which is a hybrid between the continuous and discontinuous Galerkin methods. It allows to divide the domain into subdomains on which the standard continuous finite element method is used, whereas on the interfaces between these subdomains, the interior penalty method is used, thus allowing for discontinuities. This approach allows to greatly reduce the number of additional degrees of freedom as they are only needed on the interfaces. Besides, CDGM does not require conforming grids on the interfaces, thus allowing for independent grids for the subdomains.

The composite discontinuous Galerkin method is currently successfully developed and used for various problems, for example, elliptic eigenvalue problems [8], parabolic problems [17], and Darcy flow in homogeneous porous media [16]. A FETI-DP-type method (dual primal finite element tearing and interconnecting) for CDGM in two dimensions was proposed in [7].

We aim to use the composite discontinuous Galerkin method for semiconductor device simulations due to several reasons. First, by its nature, it accounts for separate meshes on the layers of the device, so it is possible to use nonconforming grids in general and to tune the mesh for one layer without affecting the rest of the domain. Moreover, in simulations of 
gallium nitride laser diodes, the coefficients of the elliptic equations vary by several orders of magnitude at the interfaces between semiconductor material layers. This effect occurs in particular in the active region of the semiconductor devices, on interfaces between quantum wells and quantum barriers, on boundaries of the electron blocking layers, etc. Highly varying coefficients are not present in equation (1.1), but they occur in the two latter equations of (1.2). The discontinuous Galerkin method is more robust than the continuous FEM in case of discontinuous, highly variable coefficients. While in this paper we deal with equation (1.1), the goal of our study is to use the CDGM method for the van Roosbroeck system (1.2). In practice, in physical simulations, we also have to introduce additional physical effects which are not accounted for by the formulation (1.2). One important example here is polarization, which leads to significant interface charges in the nitride-based devices. This effect may be introduced into (1.1) by the addition of the distributional derivatives on the interfaces which lead to discontinuities of fluxes or unknown functions. In case of discontinuous Galerkin methods, these discontinuities may be introduced to the model in a very natural way. Another reason for using CDGM from a physical background is the local mass conservation, which is a known property of discontinuous Galerkin method [22]. This property, in our specific case, corresponds to the Gauss law, while the locality is limited to the subdomains of the device.

In this paper, we would like to present the error analysis of the CDGM variant for equilibrium state solutions of the van Roosbroeck equations in $\mathbb{R}^{2}$. We limit our analysis to this case, as the proof framework used in this paper, which is borrowed from the DGM analysis of the Navier-Stokes problem [10], imposes the uniqueness of the solution, which is not guaranteed in the non-equilibrium state. For a one-dimensional domain, we have numerical evidence of convergence of the presented method for both the equilibrium and non-equilibrium state [25]. This discretization was also used by our research group in simulations of realistic semiconductor devices [24].

In our analysis, we focus on the standard continuous polynomial $\mathbb{P}^{k}$ element. Simulations, however, are limited to the $\mathbb{P}^{1}$ case only. While there are many computer libraries and frameworks for FEM and DGM discretizations, none that we are aware of supports CDGM out of the box. In particular, it is not possible to define separate meshes across subdomains. Therefore we develop our framework currently supporting only the standard continuous linear $\mathbb{P}^{1}$ element. While a mathematical analysis is presented for equation (1.1), in simulations we also cover the full drift-diffusion system (1.2).

The remainder of this paper is organized as follows. We start with the introduction of the differential problem in Section 2. We propose a variant of the CDGM discretization of this problem in Section 3. The main result of this paper is stated in Section 4. Then we show existence and uniqueness of the introduced discrete problem in Sections 5, 6. In Section 7 we discuss interpolation properties of the discrete space. Then we pass to the error estimate in Section 8. Finally we present results of numerical simulations in Section 9, and we conclude in Section 10.

2. Differential problem. The drift-diffusion model describes the relationship between the electrostatic potential and the charge carrier concentrations: electrons and holes [27, 30]. The physical derivation of this model is beyond the scope of this work. Therefore we will focus on the mathematical aspects.

We start with the domain $\Omega$ of our problem. Luminescent semiconductor devices are made of planar layers deposited on top of each other, which vary in composition of the semiconductor material or the number of impurities (see Figure 2.1). At opposite ends, metal contacts are attached where the current can be applied. If this is the case, then it flows through the device perpendicular to the deposited layers. We assume that $\Omega$ is a rectangle with boundary $\partial \Omega=\partial \Omega_{D} \cup \partial \Omega_{N}$. 


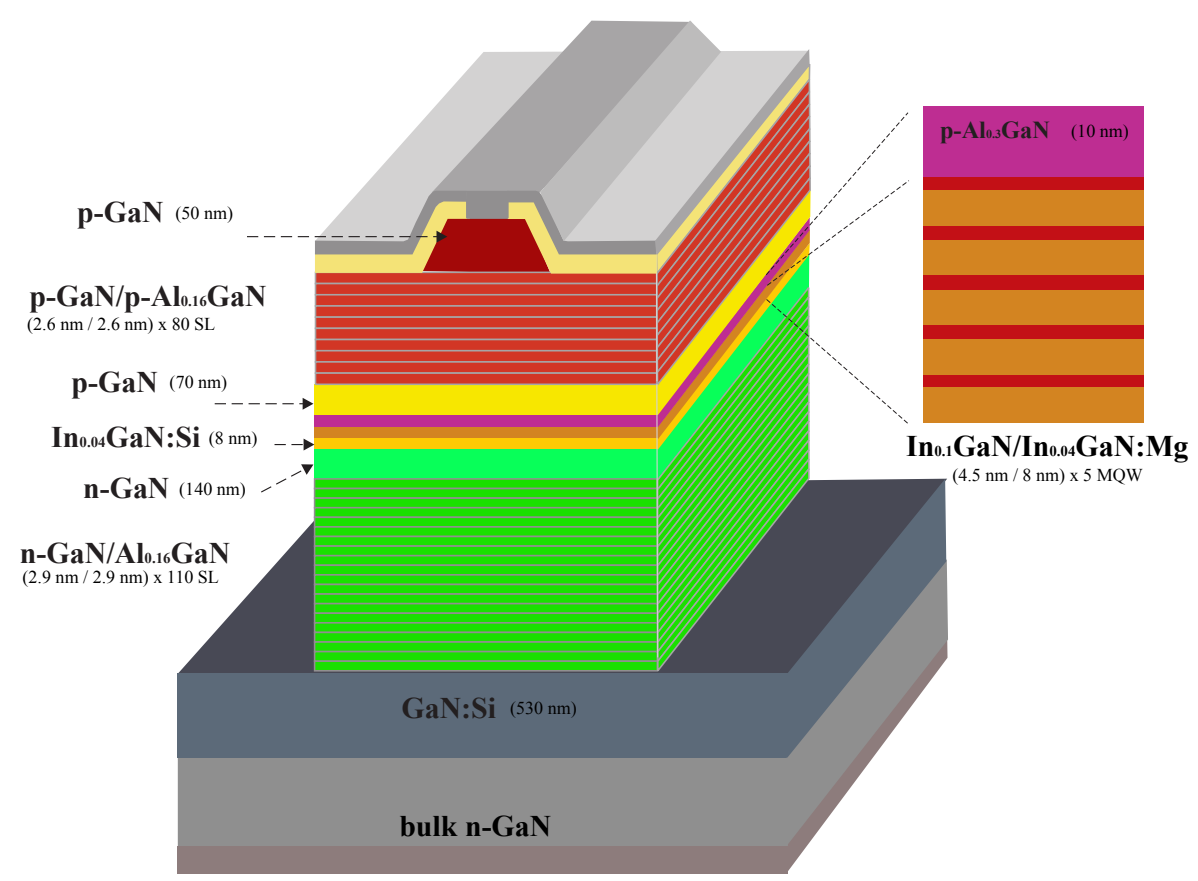

FIG. 2.1. Example of a gallium nitride semiconductor laser structure.

In this paper, we deal with the equilibrium state. It corresponds to the following differential problem: Find $u^{*} \in H^{1}(\Omega)$ such that

$$
\begin{aligned}
-\nabla \cdot\left(\varepsilon(x) \nabla u^{*}\right)+e^{u^{*}-\hat{v}}-e^{\hat{w}-u^{*}} & =k_{1}, & & \\
u^{*} & =\hat{u} & & \text { on } \partial \Omega_{D}, \\
\nabla u^{*} \cdot \nu & =0 & & \text { on } \partial \Omega_{N},
\end{aligned}
$$

where $\hat{v}=\hat{w} \equiv$ const. Since some results of this paper may be also applied to the nonequilibrium case, we consider the more general assumption that $\hat{v}, \hat{w} \in L_{\infty}(\Omega)$. Also we assume that $\varepsilon, \hat{u} \in H^{1}(\Omega) \cap L_{\infty}(\Omega)$ and $0<\varepsilon_{m} \leq \varepsilon(x) \leq \varepsilon_{M}, \varepsilon_{m}, \varepsilon_{M} \in \mathbb{R}$.

The following theorem is essential for the results presented in this paper. Its proof may be found in [12].

THEOREM 2.1. The solution $u^{*}$ of problem (2.1) is bounded.

The weak formulation of the differential problem (2.1) is as follows: Find $u^{*} \in \hat{u}+H_{0}^{1}(\Omega)$ such that

$$
a\left(u^{*}, \varphi\right)+b\left(u^{*}, \varphi\right)=f(\varphi) \quad \forall \varphi \in H_{0, \partial \Omega_{D}}^{1}(\Omega)
$$

where

$$
\begin{aligned}
a(u, \varphi) & :=\int_{\Omega} \varepsilon(x) \nabla u(x) \cdot \nabla \varphi(x) d x \\
b(u, \varphi) & :=\int_{\Omega}\left(e^{u(x)-\hat{v}(x)}-e^{\hat{w}(x)-u(x)}\right) \varphi(x) d x \\
f(\varphi) & :=\int_{\Omega} k_{1}(x) \varphi(x) d x .
\end{aligned}
$$




\begin{tabular}{|l|l|l|l|l|l|}
\hline$\Omega_{7}$ & $\Omega_{8}$ & $\Omega_{9}$ & $\Omega_{10}$ & $\Omega_{11}$ & $\Omega_{12}$ \\
\hline$\Omega_{1}$ & $\Omega_{2}$ & $\Omega_{3}$ & $\Omega_{4}$ & $\Omega_{5}$ & $\Omega_{6}$ \\
\hline
\end{tabular}

FIG. 3.1. An example of a two-dimensional coarse grid of $\Omega$.

We use the following notation:

$$
\begin{array}{r}
C_{0, \partial \Omega_{D}}^{\infty}(\bar{\Omega}):=\left\{f \in C^{\infty}(\bar{\Omega}):\left.f\right|_{\partial \Omega_{D}} \equiv 0\right\}, \\
H_{0, \partial \Omega_{D}}^{1}(\Omega):=\text { closure of } C_{0, \partial \Omega_{D}}^{\infty}(\bar{\Omega}) \text { in } H^{1}(\Omega) .
\end{array}
$$

\section{Discretization.}

3.1. Discrete space. Let $\Omega \subset \mathbb{R}^{2}$ be a rectangle divided into disjoint subrectangles $\left\{\Omega_{i}\right\}_{i=1}^{N}=: \mathcal{E}$ in such a manner that $\mathcal{E}$ is a conforming mesh [28] of $\Omega$ (Figure 3.1). We will call this division a coarse grid, and we assume that if $e \subset \partial \Omega$ is an edge of some $\Omega_{i}$, then either $e \subset \partial \Omega_{D}$ or $e \subset \partial \Omega_{N}$.

Let us define triangulations $\mathcal{T}_{h_{i}}:=\mathcal{T}_{i, h_{i}}\left(\Omega_{i}\right)$, where $h_{i}:=\max \left\{\operatorname{diam}(\tau): \tau \in \mathcal{T}_{h_{i}}\right\}$. By $\mathcal{N}_{h_{i}}$ we denote the nodes of the triangulation $\mathcal{T}_{h_{i}}$. We assume that $\left\{\mathcal{T}_{i, h_{i}}(\Omega)\right\}_{h_{i}}$ is a regular uniform family of triangulations [3]. We will define $\mathcal{T}_{h}:=\bigcup_{i=1}^{N} \mathcal{T}_{h_{i}}$. For $s>0$, we define the broken Sobolev spaces $H^{s}(\mathcal{E})$ and $H^{s}\left(\mathcal{T}_{h}\right)$ as

$$
\begin{aligned}
H^{s}(\mathcal{E}) & :=\left\{v \in L_{2}(\Omega): \forall i \in\{1, \ldots, N\} \quad v_{i}:=\left.v\right|_{\Omega_{i}} \in H^{s}\left(\Omega_{i}\right)\right\} \subset L_{2}(\Omega), \\
H^{s}\left(\mathcal{T}_{h}\right) & :=\left\{v \in L_{2}(\Omega):\left.\forall \tau \in \mathcal{T}_{h} \quad v\right|_{\tau} \in H^{s}(\tau)\right\} \subset L_{2}(\Omega) .
\end{aligned}
$$

Then on every $\Omega_{i}$, we define a discrete space $X_{h_{i}}\left(\Omega_{i}\right)$ of piecewise polynomial functions on the triangulation $\mathcal{T}_{h_{i}}$ :

$$
X_{h_{i}}:=X_{h_{i}}\left(\Omega_{i}\right):=\left\{u_{h, i} \in \mathcal{C}\left(\bar{\Omega}_{i}\right):\left.\forall \tau \in \mathcal{T}_{h_{i}} u_{h, i}\right|_{\tau} \in \mathbb{P}^{k}(\tau)\right\},
$$

where $k \geq 1$ is some integer. Finally we define $X_{h}(\Omega)$ as

$$
X_{h}(\Omega)=X_{h_{1}}\left(\Omega_{1}\right) \times \cdots \times X_{h_{N}}\left(\Omega_{N}\right) .
$$

Note that we may treat any element of $X_{h}(\Omega)$ as a piecewise-continuous function whose values are determined up to the interfaces $\partial \Omega_{i} \cap \partial \Omega_{j}$. Thus we identify $X_{h}(\Omega)$ with a suitable subset of the $L_{2}(\Omega)$ space. Then note that $X_{h}(\Omega) \not \subset H^{1}(\Omega)$ and $X_{h}(\Omega) \not \subset H^{2}(\mathcal{E})$, but $X_{h}(\Omega) \subset H^{1}(\mathcal{E}), H^{1}(\Omega) \subset H^{1}(\mathcal{E})$, and $X_{h}(\Omega) \subset H^{2}\left(\mathcal{T}_{h}\right)$.

By $\Gamma$ we denote a set of all internal and boundary edges of $\mathcal{E}$. Then $\Gamma$ is a sum of disjoint sets $\Gamma_{D}, \Gamma_{N}$, and $\Gamma_{I}$, where

$$
\Gamma_{D}:=\left\{e \in \Gamma: e \subset \partial \Omega_{D}\right\}, \quad \Gamma_{N}:=\left\{e \in \Gamma: e \subset \partial \Omega_{N}\right\}, \quad \Gamma_{I}:=\{e \in \Gamma: e \subset \operatorname{int}(\Omega)\} .
$$


Therefore $\Gamma_{D}$ (resp. $\Gamma_{N}$ ) contains edges lying on the boundary where Dirichlet (resp. Neumann) boundary conditions are imposed, and in $\Gamma_{I}$ there are all internal edges, which we call interfaces as they frequently correspond to the physical interfaces between different semiconductor materials. We also define

$$
\Gamma_{D I}:=\Gamma_{D} \cup \Gamma_{I}, \quad \Gamma_{i}:=\left\{e \in \Gamma: e \subset \partial \Omega_{i}\right\} .
$$

Let $e \in \Gamma$. Then two cases are possible. Either $e \in \Gamma_{D} \cup \Gamma_{N}$, hence there is a unique $\Omega_{i}$ such that $e$ is an edge of $\Omega_{i}$, or $e \in \Gamma_{I}$ and there are exactly two sets $\Omega_{i}, \Omega_{j} \in \mathcal{E}$ such that $e$ is their common edge. Also we define $\operatorname{nb}\left(\Omega_{i}\right):=\left\{\Omega_{l} \in \mathcal{E}: \Gamma_{i} \cap \Gamma_{l} \neq \emptyset\right\}$. Moreover, for $e \in \Gamma_{D} \cup \Gamma_{N}$, by $\nu$ we denote the normal vector to $\Omega$. On the other hand, for $e \in \Gamma_{I}, e=\partial \Omega_{i} \cap \partial \Omega_{j}, i<j$, we define $\nu$ to be a vector normal to $\Omega_{i}$. Thus also $-\nu$ is normal to $\Omega_{j}$. The opposite direction of these vectors may also be used, but they must be used consequently.

For $s>1 / 2$ we define operators

$$
[\cdot]:=[\cdot]_{e}: H^{s}(\mathcal{E}) \rightarrow L_{2}(e), \quad\{\cdot\}:=\{\cdot\}_{e}: H^{s}(\mathcal{E}) \rightarrow L_{2}(e)
$$

as

$$
\begin{gathered}
{[u]:= \begin{cases}u_{i}-u_{j} & \text { if } e \subset \Gamma_{I}, e=\partial \Omega_{i} \cap \partial \Omega_{j}, i<j, \\
u_{i} & \text { if } e \subset \Gamma_{D} \cup \Gamma_{N}, e=\partial \Omega_{i} \cap \partial \Omega,\end{cases} } \\
\{u\}:= \begin{cases}\frac{1}{2}\left(u_{i}+u_{j}\right) & \text { if } e \subset \Gamma_{I}, e=\partial \Omega_{i} \cap \partial \Omega_{j}, \\
u_{i} & \text { if } e \subset \Gamma_{D} \cup \Gamma_{N}, e=\partial \Omega_{i} \cap \partial \Omega .\end{cases}
\end{gathered}
$$

For convenience, we will also use this notion for the triangulation parameters, i.e.,

$$
\left\{h^{-s}\right\}:=\left\{\frac{1}{h^{s}}\right\}:= \begin{cases}\frac{1}{2}\left(\frac{1}{h_{i}^{s}}+\frac{1}{h_{j}^{s}}\right) & \text { if } e=\partial \Omega_{i} \cap \partial \Omega_{j}, \\ \frac{1}{h_{i}^{s}} & \text { if } e=\partial \Omega_{i} \cap \partial \Omega .\end{cases}
$$

For further analysis, we introduce the so-called broken norm $\|\cdot\|_{h}$ in $X_{h}(\Omega)$ as

$$
\left\|u_{h}\right\|_{h}^{2}:=\sum_{i=1}^{N} \int_{\Omega_{i}} \varepsilon\left(\nabla u_{h, i}\right)^{2} d x+\sum_{e \in \Gamma_{D I}} \eta_{e} \int_{e}\left[u_{h}\right]^{2} d s,
$$

where

$$
\eta_{e}:=2 \sigma_{e}\left\{h^{-1}\right\}= \begin{cases}2 \sigma_{e} h_{i}^{-1} & e \in \Gamma_{D}, e \subset \Omega_{i}, \\ \sigma_{e}\left(h_{i}^{-1}+h_{j}^{-1}\right) & e \in \Gamma_{I}, e \subset \Omega_{i} \cap \Omega_{j} .\end{cases}
$$

Here $\sigma_{e}>0$ is a penalty parameter.

To simplify the analysis, we assume that $0<\sigma_{0} \leq \sigma_{e}$ for all $e \in \Gamma_{D I}$. Also we assume that $0<h_{i}<h_{0} \leq 1$ for all $i \in\{1, \ldots, N\}$. The choice of $\sigma_{0}$ and $h_{0}$ will be discussed later in Lemmas 5.2 and 8.2.

We also need the following standard result for FEM spaces:

Lemma 3.1. For any $u_{h} \in X_{h}(\Omega), \Omega_{i} \in \mathcal{E}$, and $e \in \Gamma_{i}$, the following estimates hold:

$$
\begin{aligned}
\left\|u_{h, i}\right\|_{L_{2}(e)} & \leq C h_{i}^{-1 / 2}\left\|u_{h, i}\right\|_{L_{2}\left(\Omega_{i}\right)}, \\
\left\|\nabla u_{h, i} \cdot \nu\right\|_{L_{2}(e)} & \leq C h_{i}^{-1 / 2}\left|u_{h, i}\right|_{H^{1}\left(\Omega_{i}\right)} .
\end{aligned}
$$

The constants $C$ do not depend on $h_{i}$.

These estimates are a consequence of the trace theorem applied to each edge of fine elements in $\Omega_{i}$ coincident with $e$ followed by a scaling argument. 
3.2. Discrete problem. We discuss a variant of the composite discontinuous Galerkin discretization derived from the symmetric interior penalty Galerkin (SIPG) method (cf. [22] or [5]). We use the composite formulation (cf. [6]), i.e., inside every $\Omega_{i}$ we use the finite element method on the triangulation $\mathcal{T}_{h_{i}}$, while on the boundaries $e \in \Gamma_{D I}$ we use the discontinuous Galerkin method.

This problem is defined as follows: Find $u_{h}^{*} \in X_{h}(\Omega)$ such that

$$
a_{h}\left(u_{h}^{*}, \varphi_{h}\right)+b\left(u_{h}^{*}, \varphi_{h}\right)=f_{h}\left(u_{h}^{*}, \varphi_{h}\right), \quad \forall \varphi_{h} \in X_{h}(\Omega),
$$

where

$$
\begin{aligned}
a_{h}\left(u_{h}, \varphi_{h}\right)= & \sum_{i=1}^{N} \int_{\Omega_{i}} \varepsilon \nabla u_{h, i} \cdot \nabla \varphi_{h, i} d x-\sum_{e \in \Gamma_{D I}} \int_{e}\left\{\varepsilon \nabla u_{h} \cdot \nu\right\}\left[\varphi_{h}\right] d s \\
& -\sum_{e \in \Gamma_{D I}} \int_{e}\left\{\varepsilon \nabla \varphi_{h} \cdot \nu\right\}\left[u_{h}\right] d s+\sum_{e \in \Gamma_{D I}} \eta_{e} \int_{e}\left[u_{h}\right] \cdot\left[\varphi_{h}\right] d s \\
f_{h}\left(\varphi_{h}\right)= & \int_{\Omega} k_{1} \varphi_{h} d x-\sum_{e \in \Gamma_{D}} \int_{e}\left\{\varepsilon \nabla \varphi_{h} \cdot \nu\right\}[\hat{u}] d s \\
& +\sum_{e \in \Gamma_{D}} \eta_{e} \int_{e}[\hat{u}]\left[\varphi_{h}\right] d s,
\end{aligned}
$$

and $b(u, \varphi)$ is defined as in (2.3).

4. Main result. Most of this paper is dedicated to justifying the following result: THEOREM 4.1.

(a) The solution $u_{h}^{*} \in X_{h}(\Omega)$ of the discrete problem (3.2) exists, and it is unique.

(b) Assume that $u^{*} \in H^{1}(\Omega) \cap H^{k+1}(\mathcal{E}), k \geq 1$, is a solution of the differential problem (2.2) and $\varepsilon \in L_{\infty}(\Omega),\left.\varepsilon\right|_{\Omega_{i}} \in \mathcal{C}^{1}\left(\overline{\Omega_{i}}\right)$ for all $i \in\{1, \ldots, n\}$. Then the following error estimate holds:

$$
\begin{aligned}
\left\|u^{*}-u_{h}^{*}\right\|_{h} & \leq\left\|u^{*}-u_{I}^{*}\right\|_{h}+\left\|u_{I}^{*}-u_{h}^{*}\right\|_{h} \\
& \leq C\left(\sum_{i=1}^{N}\left(h_{i}^{2 k}+\sum_{\Omega_{l} \in \operatorname{nb}\left(\Omega_{i}\right)} \frac{h_{i}^{2 k+1}}{h_{l}}\right)\left|u_{i}^{*}\right|_{H^{k+1}\left(\Omega_{i}\right)}^{2}\right)^{1 / 2} .
\end{aligned}
$$

REMARK 4.2. If additionally we assume that $h_{i}:=c_{i} h$ for every $\Omega_{i} \in \mathcal{E}$, then the estimate (4.1) reduces to

$$
\left\|u^{*}-u_{h}^{*}\right\|_{h} \leq C h^{k}\left(\sum_{i=1}^{N}\left|u_{i}^{*}\right|_{H^{k+1}\left(\Omega_{i}\right)}^{2}\right)^{1 / 2} .
$$

Existence and uniqueness of the discrete problem are shown in Sections 5 and 6, respectively. Then the error estimate is derived in Section 8.

5. Existence. We define $P: X_{h}(\Omega) \rightarrow X_{h}^{*}(\Omega)$ as

$$
P\left(u_{h}\right) \varphi_{h}:=a_{h}\left(u_{h}, \varphi_{h}\right)+b\left(u_{h}, \varphi_{h}\right)-f_{h}\left(\varphi_{h}\right) .
$$

We would like to use the following consequence of the Brouwer theorem $[9,15]$ :

THEOREM 5.1. Let $P: X \rightarrow X^{*}$ be a continuous function on a finite-dimensional normed real vector space $X$ such that for suitable $\rho>0$ we have

$$
P(x) x \geq 0 \quad \forall\|x\| \geq \rho .
$$


Then there exists $x \in X$ such that

$$
P(x)=0 .
$$

We need the following lemma, which is a simple consequence of the Schwarz inequality and Cauchy's $\epsilon$-inequality (see also [6]):

LEMmA 5.2. There exist $\sigma_{0}>0$ and $c>0$ such that for every $\sigma_{e} \geq \sigma_{0}$ and $u_{h} \in X_{h}(\Omega)$

$$
c\left\|u_{h}\right\|_{h}^{2} \leq a_{h}\left(u_{h}, u_{h}\right) .
$$

The constant $\sigma_{0}$ depends on $\varepsilon_{m}$, $\varepsilon_{M}$, and the maximal number of edges of elements in the coarse grid $\mathcal{E}$.

Let $C:=\max \left\{\|\hat{v}\|_{L_{\infty}(\Omega)},\|\hat{w}\|_{L_{\infty}(\Omega)}\right\}$. Then we may decompose $b\left(u_{h}, u_{h}\right)$ as

$$
\begin{aligned}
b\left(u_{h}, u_{h}\right)= & \int_{\Omega}\left(e^{u_{h}-\hat{v}}-e^{\hat{w}-u_{h}}\right) u_{h} d x \\
= & \int_{\Omega}\left(e^{u_{h}-\hat{v}}-e^{\hat{w}-u_{h}}\right) u_{h} \chi_{\left\{x \in \Omega:\left|u_{h}(x)\right|>C\right\}} d x \\
& +\int_{\Omega}\left(e^{u_{h}-\hat{v}}-e^{\hat{w}-u_{h}}\right) u_{h} \chi_{\left\{x \in \Omega:\left|u_{h}(x)\right| \leq C\right\}} d x .
\end{aligned}
$$

The first integral is non-negative, and the latter we can estimate from below by

$$
\int_{\Omega}\left(e^{u_{h}(x)-\hat{v}(x)}-e^{\hat{w}(x)-u_{h}(x)}\right) u_{h}(x) \chi_{\left\{x \in \Omega:\left|u_{h}(x)\right| \leq C\right\}}(x) d x \geq-|\Omega| 2 e^{2 C} C .
$$

To estimate $f_{h}\left(u_{h}\right)$ we first use Lemma 3.1 and the trace inequality

$$
\begin{aligned}
\left|\sum_{e \in \Gamma_{D}} \int_{e}\left\{\varepsilon \nabla u_{h} \cdot \nu\right\}[\hat{u}] d s\right| & \leq \varepsilon_{M} \sum_{e \in \Gamma_{D}}\left\|\nabla u_{h}\right\|_{L_{2}(e)}\|\hat{u}\|_{L_{2}(e)} \\
& \leq c \varepsilon_{M} \sum_{i=1}^{N} h_{i}^{-1 / 2}\left\|\nabla u_{h}\right\|_{L_{2}\left(\Omega_{i}\right)}\|\hat{u}\|_{L_{2}\left(\Omega_{i}\right)} \\
& \leq C\left\|u_{h}\right\|_{h}\|\hat{u}\|_{H_{1}(\Omega)},
\end{aligned}
$$

where $C$ depends on $\varepsilon_{M}$ and $h$. Then, using the Schwarz inequality, we obtain

$$
-f_{h}\left(u_{h}\right) \geq-c\left(\hat{u}, k_{1}, h\right)\left\|u_{h}\right\|_{h} .
$$

Therefore by Lemma 5.2 and by (5.1), (5.2), we get

$$
P\left(u_{h}\right) u_{h} \geq c_{1}\left\|u_{h}\right\|_{h}^{2}-c_{2}\left\|u_{h}\right\|_{h}-c_{3},
$$

where $c_{1}, c_{2}, c_{3} \in \mathbb{R}$ are some positive constants independent of $u_{h}$. It is therefore clear that for $\left\|u_{h}\right\|_{h}$ large enough, we have that $P\left(u_{h}\right) u_{h} \geq 0$. Then by Theorem 5.1 there exists some $u_{h}^{*}$, such that $P\left(u_{h}^{*}\right)=0$.

6. Uniqueness. Assume that there exist two solutions $u_{h}^{*}, v_{h}^{*} \in X_{h}(\Omega)$ of equation (3.2). Then taking $\varphi_{h}:=u_{h}^{*}-v_{h}^{*}$ and subtracting (3.2) for $u_{h}^{*}$ and $v_{h}^{*}$ we obtain

$$
\begin{aligned}
a_{h}\left(u_{h}^{*}-v_{h}^{*}, u_{h}^{*}-v_{h}^{*}\right)= & \sum_{i=1}^{N} \int_{\Omega_{i}} e^{-\hat{v}}\left(e^{v_{h}^{*}}-e^{u_{h}^{*}}\right)\left(u_{h}^{*}-v_{h}^{*}\right) d x \\
& +\sum_{i=1}^{N} \int_{\Omega_{i}} e^{\hat{w}}\left(e^{-u_{h}^{*}}-e^{-v_{h}^{*}}\right)\left(u_{h}^{*}-v_{h}^{*}\right) d x .
\end{aligned}
$$


By the monotonicity of the exponential function, the right-hand side is nonpositive. On the other hand by Lemma 5.2 we have

$$
0<c\left\|u_{h}^{*}-v_{h}^{*}\right\|_{h}^{2} \leq a_{h}\left(u_{h}^{*}-v_{h}^{*}, u_{h}^{*}-v_{h}^{*}\right) .
$$

Thus $0<\left\|u_{h}^{*}-v_{h}^{*}\right\|_{h}^{2} \leq 0$ since $u_{h}^{*} \neq v_{h}^{*}$, and we have a contradiction.

7. Interpolation operator. For any $\Omega_{i} \in \mathcal{E}$ let $\Pi_{h_{i}}: H^{k+1}\left(\Omega_{i}\right) \rightarrow X_{h_{i}} \subset \mathcal{C}^{0}\left(\overline{\Omega_{i}}\right)$ be a standard piecewise-polynomial continuous interpolation operator. Then we define $\Pi_{h}: H^{k+1}(\mathcal{E}) \rightarrow X_{h}$ by

$$
\forall \Omega_{i} \in \mathcal{E}: \quad\left(\Pi_{h} u\right)_{i}:=\Pi_{h_{i}} u_{i} .
$$

On any $\Omega_{i}$, we can use the standard interpolation estimate for FEM [3]:

$$
\left\|u_{i}-\Pi_{h_{i}} u_{i}\right\|_{L_{2}\left(\Omega_{i}\right)}+h_{i}\left\|u_{i}-\Pi_{h_{i}} u_{i}\right\|_{H^{1}\left(\Omega_{i}\right)} \leq C h_{i}^{k+1}\left|u_{i}\right|_{H^{k+1}\left(\Omega_{i}\right)} .
$$

Let further $u_{I}:=\Pi_{h} u$.

Lemma 7.1. Let $u \in H^{k+1}(\mathcal{E}), u_{I}:=\Pi_{h} u$. For any $\Omega_{i} \in \mathcal{E}$ and for any $e \in \Gamma_{i}$,

$$
\begin{aligned}
\left\|u_{i}-u_{I, i}\right\|_{L_{2}(e)} \leq C h_{i}^{k+1 / 2}\left|u_{i}\right|_{H^{k+1}\left(\Omega_{i}\right)}, \\
\left|u_{i}-u_{I, i}\right|_{H^{1}(e)} \leq C h_{i}^{k-1 / 2}\left|u_{i}\right|_{H^{k+1}\left(\Omega_{i}\right)} .
\end{aligned}
$$

Proof. For fixed $e$ and $\Omega_{i}$, we have $\left\|u_{i}-u_{I, i}\right\|_{L_{2}(e)}^{2}=\sum_{\tau \in \mathcal{T}_{h_{i}, e}}\left\|u_{i}-u_{I, i}\right\|_{L_{2}(e \cap \tau)}^{2}$. Note that on a single triangulation element $\tau$ we have $u_{i}-u_{I, i} \in H^{2}(\tau)$, so using the trace inequality (see [22]) for $H^{2}(\tau)$ functions we have

$$
\left\|u_{i}-u_{I, i}\right\|_{L_{2}(e \cap \tau)}=C\left(h_{i}^{-1 / 2}\left\|u_{i}-u_{I, i}\right\|_{L_{2}(\tau)}+h_{i}^{1 / 2}\left|u_{i}-u_{I, i}\right|_{H_{1}(\tau)}\right) .
$$

Then by (7.1) it follows that

$$
\begin{aligned}
\left\|u_{i}-u_{I, i}\right\|_{L_{2}(e)} & \leq C h_{i}^{-1 / 2}\left(\left\|u_{i}-u_{I, i}\right\|_{L_{2}\left(\Omega_{i}\right)}+h_{i}\left\|u_{i}-u_{I, i}\right\|_{H^{1}\left(\Omega_{i}\right)}\right) \\
& \leq C h_{i}^{k+1 / 2}\left|u_{i}\right|_{H^{k+1}\left(\Omega_{i}\right)} .
\end{aligned}
$$

The proof of the latter estimate is analogous.

Let us take any $e \in \Gamma_{D I}$. For $e \in \Gamma_{I}$ we assume that $e=\Omega_{j} \cap \Omega_{l}$ for some $\Omega_{j}, \Omega_{l} \in \mathcal{E}$, and by the triangle inequality we have

$$
\int_{e}\left[u-u_{I}\right]^{2} d s=\left\|\left[u-u_{I}\right]\right\|_{L_{2}(e)}^{2} \leq 2 \sum_{i \in\{j, l\}}\left\|u_{i}-u_{I, i}\right\|_{L_{2}(e)}^{2},
$$

while for $e \in \Gamma_{D}$ we have $e \in \Gamma_{i}$ for some $\Omega_{i} \in \mathcal{E}$ and simply

$$
\int_{e}\left[u-u_{I}\right]^{2} d s=\int_{e}\left(u_{i}-u_{I, i}\right)^{2} d s=\left\|u_{i}-u_{I, i}\right\|_{L_{2}(e)}^{2} .
$$

Therefore it is sufficient to estimate $\left\|u_{i}-u_{I, i}\right\|_{L_{2}(e)}^{2}$ for any $e \in \Gamma_{D I}, e \subset \partial \Omega_{i}$ using Lemma 7.1. Let $e \in \Gamma_{D}$. By (7.2) we have

$$
\begin{aligned}
\eta_{e} \int_{e}\left(u_{i}-u_{I, i}\right)^{2} d s & =\sigma_{e} h_{i}^{-1}\left\|u_{i}-u_{I, i}\right\|_{L_{2}(e)}^{2} \\
& \leq C \sigma_{e} h_{i}^{-1} h_{i}^{2 k+1}\left|u_{i}\right|_{H^{k+1}\left(\Omega_{i}\right)}^{2}=C \sigma_{e} h_{i}^{2 k}\left|u_{i}\right|_{H^{k+1}\left(\Omega_{i}\right)}^{2}
\end{aligned}
$$


On the other hand, if $e \in \Gamma_{I}$, then

$$
\begin{aligned}
\eta_{e} \int_{e}\left(u_{j}-u_{I, j}\right)^{2} d s & =\frac{1}{2} \sigma_{e}\left(h_{j}^{-1}+h_{l}^{-1}\right)\left\|u_{j}-u_{I, j}\right\|_{L_{2}(e)}^{2} \\
& \leq C \sigma_{e}\left(h_{j}^{2 k}+\frac{h_{j}^{2 k+1}}{h_{l}}\right)\left|u_{j}\right|_{H^{k+1}\left(\Omega_{j}\right)}^{2} .
\end{aligned}
$$

Then if we sum up over $e \in \Gamma_{D I}$,

$$
\sum_{e \in \Gamma_{D I}} \eta_{e} \int_{e}\left(\left[u-u_{I}\right]\right)^{2} d s \leq \sum_{i=1}^{N} C\left(h_{i}^{2 k}+\sum_{\Omega_{l} \in \operatorname{nb}\left(\Omega_{i}\right)} \frac{h_{i}^{2 k+1}}{h_{l}}\right)\left|u_{i}\right|_{H^{k+1}\left(\Omega_{i}\right)}^{2}
$$

thus taking into account this estimate and (7.1), we obtain

$$
\left\|u-u_{I}\right\|_{h}^{2} \leq C \sum_{i=1}^{N}\left(h_{i}^{2 k}+\sum_{\Omega_{l} \in \operatorname{nb}\left(\Omega_{i}\right)} \frac{h_{i}^{2 k+1}}{h_{l}}\right)\left|u_{i}\right|_{H^{k+1}\left(\Omega_{i}\right)}^{2} .
$$

If we increase the density proportionally, i.e., $h_{i}:=c_{i} h$, then the result can be improved to

$$
\left\|u-u_{I}\right\|_{h}^{2} \leq C h^{2 k} \sum_{i=1}^{N}\left|u_{i}\right|_{H^{k+1}\left(\Omega_{i}\right)}^{2} .
$$

8. Error estimates. We start with the following auxiliary lemma.

LEMMA 8.1. Let $u \in H^{s}(\mathcal{E}), s \geq 1$. Then

$$
\|u\|_{L_{2}(\Omega)}^{2} \leq C\left[\sum_{i=1}^{N} \int_{\Omega_{i}}(\nabla u)^{2} d x+\sum_{e \in \Gamma_{I}}|e|^{-1} \int_{e}[u]^{2} d s+\sum_{e \in \Gamma_{D}} \int_{e} u^{2} d s\right] .
$$

A proof of Lemma 8.1 may be found in [2]. Next we would like to have an analog of the Poincare inequality for the $H^{s}(\mathcal{E})$ spaces.

LEMMA 8.2. Let $u \in H^{s}(\mathcal{E}), s \geq 1$. Then there exists some $h_{0}>0$ such that $\|u\|_{L_{2}(\Omega)} \leq c\|u\|_{h}$ for $0<h \leq h_{0}$, where $c$ is independent of $h$.

Proof. By definition of the broken norm (3.1), we have

$$
\|u\|_{h}^{2}:=\sum_{i=1}^{N} \int_{\Omega_{i}} \varepsilon_{i}(\nabla u)^{2} d x+\sum_{e \in \Gamma_{D I}} \eta_{e} \int_{e}[u]^{2} d s
$$

Note that $|e|$ does not depend on $h$ and $\eta_{e} \rightarrow \infty$ as $h \rightarrow 0$. Thus we can find $h_{0}>0$ such that $\eta_{e} \geq|e|^{-1}$ and $\eta_{e} \geq 1$ for any $0<h<h_{0}$, and then by Lemma 8.1

$$
\begin{aligned}
\|u\|_{L_{2}(\Omega)}^{2} & \leq C\left[\sum_{i=1}^{N} \int_{\Omega_{i}}(\nabla u)^{2} d x+\sum_{e \in \Gamma_{I}}|e|^{-1}\|[u]\|_{L_{2}(e)}^{2}+\sum_{e \in \Gamma_{D}}\|[u]\|_{L_{2}(e)}^{2}\right] \\
& \leq C\left[\varepsilon_{m}^{-1} \sum_{i=1}^{N} \int_{\Omega_{i}} \varepsilon_{i}(\nabla u)^{2} d x+\sum_{e \in \Gamma_{D I}} \eta_{e} \int_{e}[u]^{2} d s\right] \\
& \leq C_{1}\|u\|_{h}^{2} .
\end{aligned}
$$

To prove error estimates of the proposed discretization, we would like to introduce the following assumptions:

$$
u^{*} \in H^{1}(\Omega) \cap H^{k+1}(\mathcal{E}), \quad \varepsilon \in\left\{v \in L_{\infty}(\Omega):\left.\forall i \in\{1, \ldots, n\} v\right|_{\Omega_{i}} \in \mathcal{C}^{1}(\bar{\Omega})\right\} .
$$


8.1. Consistency. We start with an abstract result. Let $f \in L_{2}(\Omega)$. We pose two problems. The first is the following: Find $u^{*} \in H^{1}(\Omega)$ such that

$$
\begin{aligned}
\int_{\Omega} \varepsilon \nabla u^{*} \cdot \nabla \varphi d x & =\int_{\Omega} f \varphi d x & & \forall \varphi \in H_{0, \partial \Omega_{D}}^{1}(\Omega), \\
u^{*} & =\hat{u} & & \text { on } \partial \Omega_{D} .
\end{aligned}
$$

The second problem is posed in a broken Sobolev space: Find $u^{*} \in H^{1}(\mathcal{E})$ such that $\forall \varphi \in H^{1}(\mathcal{E}) \cap H^{2}\left(\mathcal{T}_{h}\right)$

$$
\begin{aligned}
\sum_{i=1}^{N} \int_{\Omega_{i}} \varepsilon \nabla u^{*} \cdot \nabla \varphi d x & -\sum_{e \in \Gamma_{D I}} \int_{e}\left\{\varepsilon \nabla u^{*} \cdot \nu\right\}[\varphi] d s \\
& -\sum_{e \in \Gamma_{D I}} \int_{e}\{\varepsilon \nabla \varphi \cdot \nu\}\left[u^{*}\right] d s+\sum_{e \in \Gamma_{D I}} \eta_{e} \int_{e}\left[u^{*}\right][\varphi] d s \\
= & \sum_{i=1}^{N} \int_{\Omega_{i}} f \varphi d x-\sum_{e \in \Gamma_{D}} \int_{e}\{\varepsilon \nabla \varphi \cdot \nu\}[\hat{u}] d s+\sum_{e \in \Gamma_{D}} \eta_{e} \int_{e}[\hat{u}][\varphi] d s .
\end{aligned}
$$

We would like to prove the following result.

THEOREM 8.3. Assume that the solution $u^{*}$ of problem (8.1) belongs to $H^{1}(\Omega) \cap H^{2}(\mathcal{E})$ and $\varepsilon \nabla u^{*} \in H^{1}(\mathcal{E})$. Then $u^{*}$ satisfies (8.2). Conversely, if $u^{*} \in H^{2}(\mathcal{E}) \cap H^{1}(\Omega)$ is a solution of (8.2) and $\varepsilon \nabla u^{*} \in H^{1}(\mathcal{E})$, then it is also a solution of (8.1).

The proof presented in this paper is based on the standard approach in the discontinuous Galerkin method; cf., e.g., [22].

LEMMA 8.4. Let $u \in H^{1}(\Omega) \cap H^{2}(\mathcal{E}), \varepsilon \nabla u \in\left(H^{1}(\mathcal{E})\right)^{2}, 0<\varepsilon_{m} \leq \varepsilon \leq \varepsilon_{M}$, and $f \in L_{2}(\Omega)$. The following statements are equivalent:

- $u$ satisfies

$$
\int_{\Omega} \varepsilon \nabla u \cdot \nabla \varphi=\int_{\Omega} f \varphi, \quad \forall \varphi \in H_{0, \partial \Omega_{D}}^{1}(\Omega)
$$

- $u$ satisfies

$$
\begin{aligned}
-\sum_{i=1}^{N} \int_{\Omega_{i}} \nabla \cdot\left(\varepsilon_{i} \nabla u_{i}\right) \varphi_{i} & =\int_{\Omega} f \varphi, & & \forall \varphi \in L_{2}(\Omega) \\
{\left.[\varepsilon \nabla u \cdot \nu]\right|_{e} } & =0 & & \forall e \in \Gamma_{I}, \\
\nabla u \cdot \nu & =0 & & \text { on } \partial \Omega_{N} .
\end{aligned}
$$

Proof. (8.4) $\Rightarrow$ (8.3) follows simply from Green's theorem. To prove (8.3) $\Rightarrow$ (8.4), take any $\varphi \in C_{0}^{\infty}(\Omega)$. Since $C_{0}^{\infty}(\Omega) \subset H_{0, \partial \Omega_{D}}^{1}(\Omega)$, then by (8.3) we have

$$
\int_{\Omega} \varepsilon \nabla u \cdot \nabla \varphi=\int_{\Omega} f \varphi
$$

By Green's theorem,

$$
\begin{aligned}
\int_{\Omega} f \varphi d x & =\sum_{i=1}^{N} \int_{\Omega_{i}} \varepsilon \nabla u \cdot \nabla \varphi d x \\
& =-\sum_{i=1}^{N} \int_{\Omega_{i}} \nabla \cdot(\varepsilon \nabla u) \varphi d x+\sum_{e \in \Gamma} \int_{e}[\varepsilon \nabla u \cdot \nu] \varphi d s .
\end{aligned}
$$


Since $\varphi$ is zero on $\partial \Omega$, we may rewrite the last sum as

$$
\int_{\Omega} f \varphi d x+\sum_{i=1}^{N} \int_{\Omega_{i}} \nabla \cdot(\varepsilon \nabla u) \varphi d x=\sum_{e \in \Gamma_{I}} \int_{e}[\varepsilon \nabla u \cdot \nu] \varphi d s .
$$

Note that we may treat this relationship as an equality of distributions. Since $f, \nabla \cdot(\varepsilon \nabla u) \in$ $L_{2}(\Omega)=\left(L_{2}(\Omega)\right)^{*}$, the left-hand side clearly defines a linear continuous functional over $L_{2}(\Omega)$, while the right-hand side does not unless it is identically zero, since for example it does not converge to zero provided that $\|\varphi\|_{L_{2}(\Omega)} \rightarrow 0$. Since the sum of any two elements of a conjugated space must give an element included in this space, both sides of the above equality must be zero. Thus in particular

$$
-\sum_{i=1}^{N} \int_{\Omega_{i}} \nabla \cdot(\varepsilon \nabla u) \varphi d x=\int_{\Omega} f \varphi .
$$

This statement is true for $\varphi \in C_{0}^{\infty}(\Omega)$. It is also true for any $\varphi \in L_{2}(\Omega)$ as $C_{0}^{\infty}(\Omega)$ is dense in $L_{2}(\Omega)$, and thus the first statement of (8.4) is shown.

Proof of Theorem 8.3. First we prove (8.1) $\Rightarrow$ (8.2). Assume that $u^{*}$ is a solution of (8.1) and that it belongs to $H^{1}(\Omega) \cap H^{2}(\mathcal{E})$. We have by definition

$$
\int_{\Omega} \varepsilon \nabla u^{*} \cdot \nabla \phi d x=\int_{\Omega} f \phi d x \quad \forall \phi \in H_{0, \partial \Omega_{D}}^{1}(\Omega) .
$$

We use Lemma 8.4, and we obtain that for any $\phi \in L_{2}(\Omega)$

$$
-\int_{\Omega} \nabla \cdot\left(\varepsilon \nabla u^{*}\right) \phi d x=\int_{\Omega} f \phi d x
$$

Let us take any $\varphi \in H^{1}(\mathcal{E}) \cap H^{2}\left(\mathcal{T}_{h}\right)$ and substitute $\phi:=\varphi$. We may split the integrals into

$$
-\sum_{i=1}^{N} \int_{\Omega_{i}} \nabla \cdot\left(\varepsilon \nabla u^{*}\right) \varphi d x=\sum_{i=1}^{N} \int_{\Omega_{i}} f \varphi d x
$$

By Green's theorem, we have

$$
-\int_{\Omega_{i}} \nabla \cdot\left(\varepsilon \nabla u^{*}\right) \varphi d x=\int_{\Omega_{i}} \varepsilon \nabla u^{*} \cdot \nabla \varphi d x-\int_{\partial \Omega_{i}} \varepsilon \nabla u^{*} \cdot \nu \varphi d x .
$$

Summing up these results in $\Omega_{i}$, we get

$$
\sum_{i=1}^{N} \int_{\Omega_{i}} \varepsilon \nabla u^{*} \cdot \nabla \varphi d x-\sum_{i=1}^{N} \int_{\partial \Omega_{i}} \varepsilon \nabla u^{*} \cdot \nu \varphi d x=\sum_{i=1}^{N} \int_{\Omega_{i}} f \varphi d x .
$$

By Lemma 8.4, we have that $\left[\varepsilon \nabla u^{*}\right]=0$ on every $e \in \Gamma_{I}$, thus $\left\{\varepsilon \nabla u^{*} \cdot \nu\right\}=\varepsilon \nabla u^{*} \cdot \nu$ on any $\partial \Omega_{i}$, and we have

$$
\sum_{i=1}^{N} \int_{\Omega_{i}} \varepsilon \nabla u^{*} \cdot \nabla \varphi d x-\sum_{e \in \Gamma} \int_{e}\left\{\varepsilon \nabla u^{*} \cdot \nu\right\}[\varphi] d x=\sum_{i=1}^{N} \int_{\Omega_{i}} f \varphi d x .
$$


By the homogeneous Neumann boundary condition (Lemma 8.4) on $e \in \Gamma_{N}$ we have $\left\{\varepsilon \nabla u^{*} \cdot \nu\right\}=0$ and

$$
\sum_{i=1}^{N} \int_{\Omega_{i}} \varepsilon \nabla u^{*} \cdot \nabla \varphi d x-\sum_{e \in \Gamma_{D I}} \int_{e}\left\{\varepsilon \nabla u^{*} \cdot \nu\right\}[\varphi] d x=\sum_{i=1}^{N} \int_{\Omega_{i}} f \varphi d x .
$$

Since $u^{*} \in H^{1}(\Omega)$, then $\left[u^{*}\right]=0$ for any $e \in \Gamma_{I}$, and by the assumption on $e \in \Gamma_{D}$ we have $u^{*}=\hat{u}$, thus we have for any $\varphi \in H^{1}(\mathcal{E})$

$$
\begin{aligned}
\sum_{e \in \Gamma_{D I}} \eta_{e} \int_{e}\left[u^{*}\right][\varphi] d s & -\sum_{e \in \Gamma_{D I}} \int_{e}\{\varepsilon \nabla \varphi \cdot \nu\}\left[u^{*}\right] d s \\
& =\sum_{e \in \Gamma_{D}} \eta_{e} \int_{e}[\hat{u}][\varphi] d s-\sum_{e \in \Gamma_{D}} \int_{e}\{\varepsilon \nabla \varphi \cdot \nu\}[\hat{u}] d s .
\end{aligned}
$$

By adding this result side-by-side to (8.5) we obtain (8.2).

We proceed to $(8.2) \Rightarrow(8.1)$. Assume that (8.2) is true. First, we recover the Dirichlet boundary conditions. Take any $e \in \Gamma_{D}$ such that $e \in \partial \Omega_{i}$ and $\bar{\varphi} \in C_{0}^{\infty}(e)$. Then let $\left\{\varphi_{\epsilon}\right\}_{\epsilon}$ be a sequence of functions, such that

$$
\begin{aligned}
& \varphi_{\epsilon} \in C^{\infty}(\Omega),\left.\quad \varphi_{\epsilon}\right|_{e}=\bar{\varphi}, \quad \operatorname{supp}\left(\varphi_{\epsilon}\right) \subset \Omega_{i} \cup e, \\
& \left.\varphi_{\epsilon}\right|_{\partial \Omega_{i} \backslash e} \equiv 0,\left.\quad \nabla \varphi_{\epsilon} \cdot \nu\right|_{\partial \Omega_{i}}=0, \quad\left\|\varphi_{\epsilon}\right\|_{L_{2}(\Omega)} \underset{\epsilon \rightarrow 0}{\longrightarrow} 0 .
\end{aligned}
$$

Then $\varphi \in H^{1}(\mathcal{E}) \cap H^{2}\left(\mathcal{T}_{h}\right)$ and (8.2) becomes

$$
\int_{\Omega_{i}} \varepsilon \nabla u^{*} \cdot \nabla \varphi_{\epsilon} d x-\int_{e} \varepsilon \nabla u^{*} \cdot \nu \bar{\varphi} d s+\eta_{e} \int_{e} u^{*} \bar{\varphi} d s=\int_{\Omega_{i}} f \varphi_{\epsilon} d x+\eta_{e} \int_{e} \hat{u} \bar{\varphi} d s .
$$

By Green's theorem,

$$
\int_{\Omega_{i}} \nabla \cdot\left(\varepsilon \nabla u^{*}\right) \varphi_{\epsilon} d x+\eta_{e} \int_{e} u^{*} \bar{\varphi} d s=\int_{\Omega_{i}} f \varphi_{\epsilon} d x+\eta_{e} \int_{e} \hat{u} \bar{\varphi} d s .
$$

Passing to the limit $\epsilon \rightarrow 0$,

$$
\eta_{e} \int_{e} u^{*} \bar{\varphi} d s=\eta_{e} \int_{e} \hat{u} \bar{\varphi} d s
$$

Since $\bar{\varphi} \in C_{0}^{\infty}(e)$ and $e \in \Gamma_{D}$ are arbitrary, we get

$$
\left.u^{*}\right|_{\partial \Omega_{D}}=\left.\hat{u}\right|_{\partial \Omega_{D}}
$$

and the Dirichlet boundary conditions are satisfied.

Take any $\varphi \in C_{0, \partial \Omega_{D}}^{\infty}(\bar{\Omega})$. Thus,

$$
\sum_{e \in \Gamma_{D I}} \eta_{e} \int_{e}\left[u^{*}\right][\varphi] d s=\sum_{e \in \Gamma_{D}} \eta_{e} \int_{e}[\hat{u}][\varphi] d s=0
$$

as $[\varphi]=0$ for any $e \in \Gamma_{I}$ since $\varphi \in C_{0, \partial \Omega_{D}}^{\infty}(\bar{\Omega})$, and on $e \in \Gamma_{D}$ we have $[\varphi]=\varphi \equiv 0$. Analogously, we see that

$$
-\sum_{e \in \Gamma_{D I}} \int_{e}\left\{\varepsilon \nabla u^{*} \cdot \nu\right\}[\varphi] d s=0
$$


By the assumptions of the theorem $u^{*} \in H^{1}(\Omega)$, so $\left[u^{*}\right]=0$ for any $e \in \Gamma_{I}$. Also, we have already shown that $u^{*}=\hat{u}$ for $e \in \Gamma_{D}$, so

$$
-\sum_{e \in \Gamma_{D I}} \int_{e}\{\varepsilon \nabla \varphi \cdot \nu\}\left[u^{*}\right] d s=-\sum_{e \in \Gamma_{D}} \int_{e}\{\varepsilon \nabla \varphi \cdot \nu\}[\hat{u}] d s .
$$

Thus, we obtain

$$
\sum_{i=1}^{N} \int_{\Omega_{i}} \varepsilon \nabla u^{*} \cdot \nabla \varphi d x=\int_{\Omega} f \varphi d x .
$$

Since this statement is true for any $\varphi \in C_{0, \partial \Omega_{D}}^{\infty}(\bar{\Omega})$, it is valid for any $\varphi \in H_{0, \partial \Omega_{D}}^{1}(\Omega)$ as well, so we regain the first statement of (8.1).

8.2. Auxiliary estimates. For better readability, we will divide the differential operators into several components. We define the following operators

$$
\begin{aligned}
A(u, \varphi) & :=\sum_{i=1}^{N} \int_{\Omega_{i}} \varepsilon \nabla u \cdot \nabla \varphi d x, \\
B(u, \varphi) & :=\sum_{i=1}^{N} \int_{\Omega_{i}}\left(e^{u-\hat{v}}-e^{\hat{w}-u}\right) \varphi d x, \\
C(\varphi) & :=\sum_{i=1}^{N} \int_{\Omega_{i}} k_{1} \varphi d x, \\
D(u, \varphi) & :=-\sum_{e \in \Gamma_{D I}} \int_{e}\left\{\varepsilon \frac{\partial u}{\partial \nu}\right\}[\varphi] d s, \\
E(u, \varphi) & :=-\sum_{e \in \Gamma_{D I}} \int_{e}\left\{\varepsilon \frac{\partial \varphi}{\partial \nu}\right\}[u] d s, \\
F(\varphi) & :=-\sum_{e \in \Gamma_{D}} \int_{e}\{\varepsilon \nabla \varphi \cdot n\}[\hat{u}] d s, \\
I(\varphi) & :=\sum_{e \in \Gamma_{D}} \eta_{e} \int_{e}[\hat{u}] \cdot[\varphi] d s, \\
J(u, \varphi) & :=\sum_{e \in \Gamma_{D I}} \eta_{e} \int_{e}[u] \cdot[\varphi] d s .
\end{aligned}
$$

In this section, we will prove several estimates for these operators. These estimates will be used in the derivation of the main result in Section 8.3.

LEMMA 8.5. Let $u_{h} \in X_{h}(\Omega)$. Then

$$
A\left(u_{h}, u_{h}\right)+J\left(u_{h}, u_{h}\right)+D\left(u_{h}, u_{h}\right)+E\left(u_{h}, u_{h}\right) \geq c\left\|u_{h}\right\|_{h}^{2} .
$$

Proof. It is a simple consequence of Lemma 5.2.

Lemma 8.6. Let $u, v \in L_{2}(\Omega)$. Then $B(u, u-v)-B(v, u-v) \geq 0$.

Proof. Since the exponential function is monotone, we have

$$
\begin{aligned}
B(u, u-v)-B(v, u-v)= & \int_{\Omega} e^{-\hat{v}}\left(e^{u}-e^{v}\right)(u-v) d x \\
& \quad+\int_{\Omega} e^{\hat{w}}\left(e^{-v}-e^{-u}\right)(u-v) d x \geq 0 .
\end{aligned}
$$


Lemma 8.7. Let $u, \varphi \in H^{1}(\mathcal{E})$. Then

$$
|A(u, \varphi)+J(u, \varphi)| \leq C\|u\|_{h}\|\varphi\|_{h} .
$$

Proof. It is a simple consequence of the Schwarz inequality.

Lemma 8.8. Let $u, v, \varphi \in H^{1}(\mathcal{E})$ and $\alpha \leq u, v \leq \beta$ for some $\alpha, \beta \in \mathbb{R}$. Then

$$
|B(u, \varphi)-B(v, \varphi)| \leq C\|u-v\|_{h}\|\varphi\|_{h},
$$

where $C$ is a constant dependent on $\alpha, \beta,\|\hat{v}\|_{L_{\infty}(\Omega)}$, and $\|\hat{w}\|_{L_{\infty}(\Omega)}$.

Proof. Note that the exponential function is locally Lipschitz-continuous, so since $u, v$ are bounded,

$$
\left\|e^{u}-e^{v}\right\|_{L_{2}(\Omega)} \leq C\|u-v\|_{L_{2}(\Omega)} .
$$

The same is true for $e^{-v}-e^{-u}$. Thus using the Schwarz inequality and the Poincaré inequality for the broken norm (Lemma 8.2), we obtain

$$
\begin{aligned}
|B(u, \varphi)-B(v, \varphi)| & =\left|\int_{\Omega} e^{-\hat{v}}\left(e^{u}-e^{v}\right) \varphi d x+\int_{\Omega} e^{\hat{w}}\left(e^{-v}-e^{-u}\right) \varphi d x\right| \\
& \leq C\|u-v\|_{L_{2}(\Omega)}\|\varphi\|_{L_{2}(\Omega)} \leq C\|u-v\|_{h}\|\varphi\|_{h} .
\end{aligned}
$$

Lemma 8.9. Let $u \in H^{k+1}(\mathcal{E}), u_{I}:=\Pi_{h} u$ (see Section 7) and $\varphi_{h} \in X_{h}(\Omega)$. Then

$$
\left|D\left(u-u_{I}, \varphi_{h}\right)\right| \leq C h^{k}\left(\sum_{i=1}^{N}\left|u_{i}\right|_{H^{k+1}\left(\Omega_{i}\right)}^{2}\right)^{1 / 2}\left\|\varphi_{h}\right\|_{h} .
$$

The constant $C$ depends on $\varepsilon_{M}$ and $\sigma_{0}$.

Proof. We have

$$
D\left(u-u_{I}, \varphi_{h}\right)=-\sum_{e \in \Gamma_{D I}} \int_{e}\left\{\varepsilon \nabla\left(u-u_{I}\right) \cdot \nu\right\}\left[\varphi_{h}\right] d s .
$$

Let us take any $e \in \Gamma_{I}, e \in \partial \Omega_{j} \cap \partial \Omega_{l}$. Then the Schwarz inequality yields that

$$
\int_{e}\left\{\varepsilon \nabla\left(u-u_{I}\right) \cdot \nu\right\}\left[\varphi_{h}\right] d s \leq \varepsilon_{M}\left\|\left\{\nabla\left(u-u_{I}\right) \cdot \nu\right\}\right\|_{L_{2}(e)}\left\|\left[\varphi_{h}\right]\right\|_{L_{2}(e)} .
$$

Then by Lemma 7.1 we get

$$
\begin{aligned}
\left\|\left\{\nabla\left(u-u_{I}\right) \cdot \nu\right\}\right\|_{L_{2}(e)} & \leq\left(h_{j}^{k-1 / 2}\left|u_{j}\right|_{H^{k+1}\left(\Omega_{j}\right)}+h_{l}^{k-1 / 2}\left|u_{l}\right|_{H^{k+1}\left(\Omega_{l}\right)}\right) \\
& \leq\left(h_{j}+h_{l}\right)^{k-1 / 2}\left(\left|u_{j}\right|_{H^{k+1}\left(\Omega_{j}\right)}+\left|u_{l}\right|_{H^{k+1}\left(\Omega_{l}\right)}\right) .
\end{aligned}
$$

Therefore,

$$
\begin{aligned}
\eta_{e}^{-1}\left\|\left\{\nabla\left(u-u_{I}\right) \cdot \nu\right\}\right\|_{L_{2}(e)}^{2} & \leq C \sigma_{e}^{-1} h_{j} h_{l}\left(h_{j}+h_{l}\right)^{2 k-2}\left(\left|u_{j}\right|_{H^{k+1}\left(\Omega_{j}\right)}+\left|u_{l}\right|_{H^{k+1}\left(\Omega_{l}\right)}\right)^{2} \\
& \leq C h^{2 k}\left(\left|u_{j}\right|_{H^{k+1}\left(\Omega_{j}\right)}+\left|u_{l}\right|_{H^{k+1}\left(\Omega_{l}\right)}\right)^{2} .
\end{aligned}
$$

If $e \in \Gamma_{D}, e \in \partial \Omega_{i}$, then analogously we have

$$
\eta_{e}^{-1}\left\|\left\{\nabla\left(u-u_{I}\right) \cdot \nu\right\}\right\|_{L_{2}(e)}^{2} \leq C \sigma_{e}^{-1} h_{i}^{2 k}\left|u_{i}\right|_{H^{k+1}\left(\Omega_{i}\right)}^{2} \leq C h^{2 k}\left|u_{i}\right|_{H^{k+1}\left(\Omega_{i}\right)}^{2} .
$$


Therefore by the Schwarz inequality and the inequalities derived above, we conclude that

$$
\begin{aligned}
& \sum_{e \in \Gamma_{D I}} \int_{e}\left\{\varepsilon \nabla\left(u-u_{I}\right) \cdot \nu\right\}\left[\varphi_{h}\right] d s \\
& \quad \leq C\left(\sum_{e \in \Gamma_{D I}} \eta_{e}^{-1}\left\|\left\{\nabla\left(u-u_{I}\right) \cdot \nu\right\}\right\|_{L_{2}(e)}^{2}\right)^{1 / 2}\left(\sum_{e \in \Gamma_{D I}} \eta_{e}\left\|\left[\varphi_{h}\right]\right\|_{L_{2}(e)}^{2}\right)^{1 / 2} \\
& \quad \leq C h^{k}\left(\sum_{i=1}^{N}\left|u_{i}\right|_{H^{k+1}\left(\Omega_{i}\right)}^{2}\right)^{1 / 2}\left\|\varphi_{h}\right\|_{h} .
\end{aligned}
$$

The constant $C$ is independent of $h$. It depends on $\sigma_{0}, \varepsilon_{M}$, and on the number of elements of $\Gamma_{D I}$.

Lemma 8.10. Let $u \in H^{2}(\mathcal{E}), u_{I}:=\Pi_{h} u$ (see Section 7), and $\varphi_{h} \in X_{h}(\Omega)$. Then

$$
\left|E\left(u-u_{I}, \varphi_{h}\right)\right| \leq C\left\|\varphi_{h}\right\|_{h}\left[\sum_{i=1}^{N}\left(h_{i}^{2 k}+\sum_{\Omega_{l} \in \operatorname{nb}\left(\Omega_{i}\right)} \frac{h_{i}^{2 k+1}}{h_{l}}\right)\left|u_{i}\right|_{H^{k+1}\left(\Omega_{i}\right)}^{2}\right]^{1 / 2} .
$$

Proof. By the Schwarz inequality,

$$
\begin{aligned}
\left|E\left(u-u_{I}, \varphi_{h}\right)\right| & \leq \sum_{e \in \Gamma_{D I}} \int_{e}\left|\left\{\varepsilon \nabla \varphi_{h} \cdot \nu\right\}\right|\left|\left[u-u_{I}\right]\right| d s \\
& \leq \varepsilon_{M} \sum_{e \in \Gamma_{D I}}\left\|\left\{\nabla \varphi_{h} \cdot \nu\right\}\right\|_{L_{2}(e)}\left\|\left[u-u_{I}\right]\right\|_{L_{2}(e)} .
\end{aligned}
$$

Splitting this sum up we get

$$
\left\|\left\{\nabla \varphi_{h} \cdot \nu\right\}\right\|_{L_{2}(e)} \leq\left\|\left.\nabla \varphi_{h} \cdot \nu\right|_{\Omega_{i}}\right\|_{L_{2}(e)}+\left\|\left.\nabla \varphi_{h} \cdot \nu\right|_{\Omega_{l}}\right\|_{L_{2}(e)} .
$$

Then using Lemma 3.1 we have

$$
\left\|\left.\nabla \varphi_{h} \cdot \nu\right|_{\Omega_{i}}\right\|_{L_{2}(e)}^{2} \leq C h_{i}^{-1}\left\|\nabla \varphi_{h}\right\|_{L_{2}\left(\Omega_{i}\right)}^{2} \leq C h_{i}^{-1}\left\|\varphi_{h}\right\|_{h}^{2} .
$$

On the other hand we see that

$$
\left\|\left[u-u_{I}\right]\right\|_{L_{2}(e)} \leq\left\|u-\left.u_{I}\right|_{\Omega_{i}}\right\|_{L_{2}(e)}+\left\|u-\left.u_{I}\right|_{\Omega_{l}}\right\|_{L_{2}(e)} .
$$

By Lemma 7.1 we have $\left\|u-\left.u_{I}\right|_{\Omega_{i}}\right\|_{L_{2}(e)}^{2} \leq C h_{i}^{2 k+1}|u|_{H^{k+1}\left(\Omega_{i}\right)}^{2}$, so

$$
\left|E\left(u-u_{I}, \varphi_{h}\right)\right| \leq C\left\|\varphi_{h}\right\|_{h}\left[\sum_{i=1}^{N}\left(h_{i}^{2 k}+\sum_{\Omega_{l} \in \operatorname{nb}\left(\Omega_{i}\right)} \frac{h_{i}^{2 k+1}}{h_{l}}\right)\left|u_{i}\right|_{H^{k+1}\left(\Omega_{i}\right)}^{2}\right]^{1 / 2} .
$$

8.3. Main estimate. The differential problem (8.2) satisfies:

$$
\begin{aligned}
A\left(u^{*}, \varphi\right)+B\left(u^{*}, \varphi\right)+D\left(u^{*}, \varphi\right) & +E\left(u^{*}, \varphi\right)+J\left(u^{*}, \varphi\right) \\
= & C(\varphi)+F(\varphi)+I(\varphi) \quad \forall \varphi \in H^{1}(\mathcal{E}) \cap H^{2}\left(\mathcal{T}_{h}\right) .
\end{aligned}
$$

On the other hand, the family of discrete problems depending on the parameter $h$ is defined as

$$
\begin{aligned}
A\left(u_{h}^{*}, \varphi_{h}\right)+B\left(u_{h}^{*}, \varphi_{h}\right)+D\left(u_{h}^{*}, \varphi_{h}\right)+E\left(u_{h}^{*}, \varphi_{h}\right)+J\left(u_{h}^{*}, \varphi_{h}\right) & \\
& =C\left(\varphi_{h}\right)+F\left(\varphi_{h}\right)+I\left(\varphi_{h}\right) \quad \forall \varphi_{h} \in X_{h} .
\end{aligned}
$$


We subtract these equations from each other with $\varphi:=\varphi_{h}$, and we obtain

$$
\begin{aligned}
A\left(u^{*}-u_{h}^{*}, \varphi_{h}\right)+B\left(u^{*}, \varphi_{h}\right) & -B\left(u_{h}^{*}, \varphi_{h}\right)+D\left(u^{*}-u_{h}^{*}, \varphi_{h}\right) \\
& +E\left(u^{*}-u_{h}^{*}, \varphi_{h}\right)+J\left(u^{*}-u_{h}^{*}, \varphi_{h}\right)=0 .
\end{aligned}
$$

This is equivalent to LHS $=$ RHS, where

$$
\begin{aligned}
\text { LHS }:= & A\left(u_{I}^{*}-u_{h}^{*}, \varphi_{h}\right)+B\left(u_{I}^{*}, \varphi_{h}\right)-B\left(u_{h}^{*}, \varphi_{h}\right)+D\left(u_{I}^{*}-u_{h}^{*}, \varphi_{h}\right) \\
& +E\left(u_{I}^{*}-u_{h}^{*}, \varphi_{h}\right)+J\left(u_{I}^{*}-u_{h}^{*}, \varphi_{h}\right),
\end{aligned}
$$

and

$$
\begin{aligned}
\text { RHS }:= & A\left(u_{I}^{*}-u^{*}, \varphi_{h}\right)+B\left(u_{I}^{*}, \varphi_{h}\right)-B\left(u^{*}, \varphi_{h}\right)+D\left(u_{I}^{*}-u^{*}, \varphi_{h}\right) \\
& +E\left(u_{I}^{*}-u^{*}, \varphi_{h}\right)+J\left(u_{I}^{*}-u^{*}, \varphi_{h}\right) .
\end{aligned}
$$

Let us take $\varphi_{h}:=u_{I}^{*}-u_{h}^{*}$. Then Lemma 8.5 and Lemma 8.6 imply the lower estimate LHS $\geq c\left\|u_{I}^{*}-u_{h}^{*}\right\|_{h}^{2}$. Also we may estimate RHS with Lemmas 8.7, 8.8, 8.9, and 8.10 as

$$
\mathrm{RHS} \leq C\left\|u_{I}^{*}-u_{h}^{*}\right\|_{h}\left(\left\|u_{I}^{*}-u^{*}\right\|_{h}+\left[\sum_{i=1}^{N}\left(h_{i}^{2 k}+\sum_{\Omega_{l} \in \operatorname{nb}\left(\Omega_{i}\right)} \frac{h_{i}^{2 k+1}}{h_{l}}\right)\left|u_{i}^{*}\right|_{H^{k+1}\left(\Omega_{i}\right)}^{2}\right]^{1 / 2}\right) .
$$

Thus estimating LHS $=$ RHS from below and above and dividing by $\left\|u_{I}^{*}-u^{*}\right\|_{h}>0$, we obtain

$$
\left\|u_{I}^{*}-u_{h}^{*}\right\|_{h} \leq C\left(\left\|u_{I}^{*}-u^{*}\right\|_{h}+\left[\sum_{i=1}^{N}\left(h_{i}^{2 k}+\sum_{\Omega_{l} \in \operatorname{nb}\left(\Omega_{i}\right)} \frac{h_{i}^{2 k+1}}{h_{l}}\right)\left|u_{i}^{*}\right|_{H^{k+1}\left(\Omega_{i}\right)}^{2}\right]^{1 / 2}\right) .
$$

Thus by the triangle inequality and the interpolation error estimate (7.3) we have

$$
\begin{aligned}
\left\|u^{*}-u_{h}^{*}\right\|_{h} & \leq\left\|u^{*}-u_{I}^{*}\right\|_{h}+\left\|u_{I}^{*}-u_{h}^{*}\right\|_{h} \\
& \leq C\left(\sum_{i=1}^{N}\left(h_{i}^{2 k}+\sum_{\Omega_{l} \in \operatorname{nb}\left(\Omega_{i}\right)} \frac{h_{i}^{2 k+1}}{h_{l}}\right)\left|u_{i}^{*}\right|_{H^{k+1}\left(\Omega_{i}\right)}^{2}\right)^{1 / 2} .
\end{aligned}
$$

Theorem 4.1 is therefore proven. For Remark 4.2, we assume that $h_{i}:=c_{i} h$ for every $\Omega_{i} \in \mathcal{E}$, and this estimate simplifies to

$$
\left\|u^{*}-u_{h}^{*}\right\|_{h} \leq C h^{k}\left(\sum_{i=1}^{N}\left|u_{i}^{*}\right|_{H^{k+1}\left(\Omega_{i}\right)}^{2}\right)^{1 / 2} .
$$

9. Numerical experiments. We would like to test whether the error estimate derived in Section 8 can be observed in numerical simulations. Therefore we present two examples. These examples are not directly related to any specific semiconductor material. Simulations of realistic semiconductor devices require accounting for material parameters and physical phenomena. These modifications do not substantially change the van Roosbroeck equations, but they go beyond the simplified model considered in this study.

Thus we will present simulations of abstract devices mimicking semiconductor p-n diodes. Our first example is a device which consists of two layers $\Omega_{1}, \Omega_{2}$, corresponding to an $\mathrm{n}$-type layer and a p-type layer of the p-n diode (Figure 9.1). It has two contacts with metal electrodes, left and right, denoted by $\partial \Omega_{D, 1}$ and $\partial \Omega_{D, 2}$. Horizontal boundaries correspond to the contact with an insulator (e.g., air). Parameters of the device are presented in Table 9.1. We control 


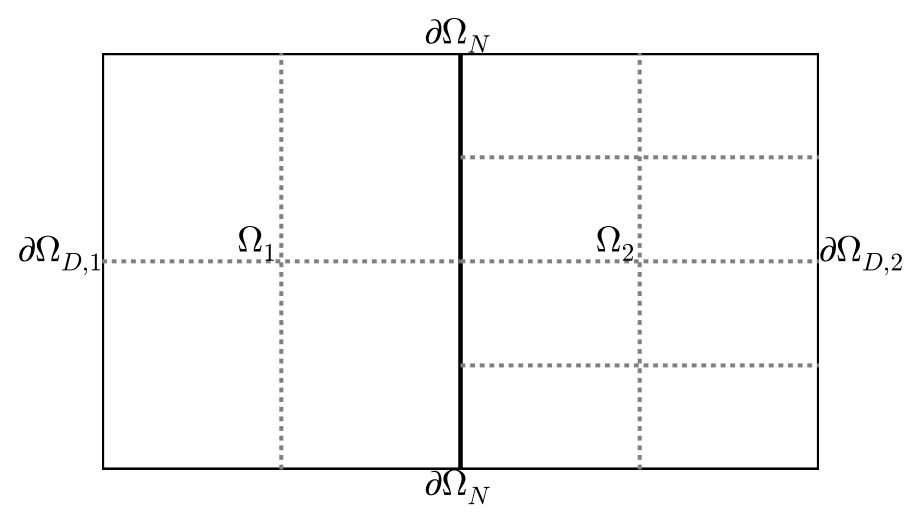

FIG. 9.1. Schema of the first device used in the simulations. It has two layers, corresponding to an n-type layer $\Omega_{1}$ and a p-type layer $\Omega_{2}$. The grid for $K=1$ is presented.

TABLE 9.1

Parameters of the first device used in the simulations. $N_{x}$ and $N_{y}$ denote the number of nodes in horizontal and vertical direction depending on the parameter $K$.

\begin{tabular}{lcc} 
Param. & $\Omega_{1}$ & $\Omega_{2}$ \\
\hline Length & $1 \times 10^{-2}$ & $1 \times 10^{-2}$ \\
Width & $1 \times 10^{-2}$ & $1 \times 10^{-2}$ \\
$N_{x}$ & $2 K+1$ & $2 K+1$ \\
$N_{y}$ & $2 K+1$ & $4 K+1$ \\
$\varepsilon$ & $3 \times 10^{-3}$ & $1 \times 10^{-3}$ \\
$\mu_{n}$ & $1 \times 10^{3}$ & $3 \times 10^{3}$ \\
$\mu_{p}$ & $1 \times 10^{2}$ & $3 \times 10^{2}$ \\
$k_{1}$ & $3 \times 10^{2}$ & $-3 \times 10^{2}$ \\
$C_{\text {rad }}$ & $1 \times 10^{-3}$ & $2 \times 10^{-3}$
\end{tabular}

the thickness of the grid with the parameter $K$. For $K=1$, we divide both layers into two pieces in the horizontal direction, while in the vertical direction, $\Omega_{1}$ is divided into two and $\Omega_{2}$ into four pieces (see Figure 9.1). The grid nodes are distributed uniformly in horizontal and vertical direction within a given $\Omega_{i}$, and their number depends on the parameter $K$ as indicated by the parameters $N_{x}, N_{y}$ in Table 9.1.

In these simulations we assume that the operator $Q$ of equation (1.2) is some given piecewise-constant function,

$$
Q(x, u, v, w):=C_{\mathrm{rad}}(x) .
$$

This form corresponds to the radiative recombination [26]. This physical process is responsible for emitting the light by a device.

We start with the equilibrium state. Then the boundary conditions are as follows: $\left.\hat{u}\right|_{\partial \Omega_{D, 1}}=0$ and $\left.\hat{u}\right|_{\partial \Omega_{D, 2}}=u_{\text {built }}$, where $u_{\text {built }}$ is called a built-in potential. It is chosen such that the charge defined as

$$
\rho(x):=k_{1}(x)-n(x)+p(x)
$$

is zero on $\partial \Omega_{D, 2}$ if $u \equiv u_{\text {built. Here }} n, p$ are the concentration of electrons and the concentration of holes, defined as

$$
n(x):=e^{u(x)-v(x)}, \quad p(x):=e^{w(x)-u(x)} .
$$




\section{ETNA}

Kent State University and

Johann Radon Institute (RICAM)

TABLE 9.2

$L_{2}(\Omega)$ - and $H^{1}(\Omega)$-error of $u$ as a function of the grid density parameter $K$ for the first device in an equilibrium state. Numbers in brackets denote the error norm reduction factor.

\begin{tabular}{rrrrr}
$\mathrm{K}$ & \multicolumn{2}{c}{$L_{2}(\Omega)$} & \multicolumn{2}{c}{$H^{1}(\Omega)$} \\
\hline 1 & $4.6 \times 10^{-2}$ & & $3.0 \times 10^{-1}$ & \\
2 & $1.1 \times 10^{-2}$ & $(4.0)$ & $1.5 \times 10^{-1}$ & $(2.0)$ \\
4 & $2.9 \times 10^{-3}$ & $(4.0)$ & $7.6 \times 10^{-2}$ & $(2.0)$ \\
8 & $6.9 \times 10^{-4}$ & $(4.1)$ & $3.7 \times 10^{-2}$ & $(2.0)$ \\
16 & $1.5 \times 10^{-4}$ & $(4.7)$ & $1.7 \times 10^{-2}$ & $(2.2)$
\end{tabular}

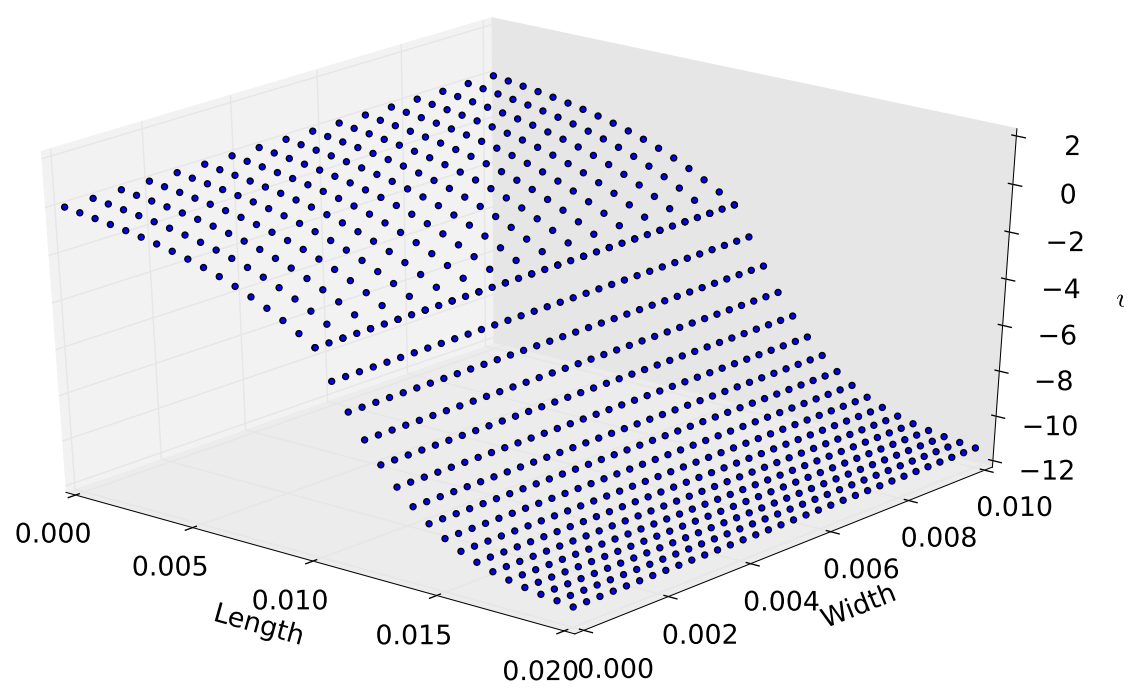

FIG. 9.2. The function $u$ for the first example in the equilibrium state for $K=8$. Note the one-dimensional character of the solution.

This is a standard choice of the boundary conditions for the equilibrium state, and it is motivated by physical arguments [26]. The functions $v, w$ are constant such that $\left.\rho\right|_{\partial \Omega_{D, 1}}=0$.

Simulations were performed for $K \in\{1,2,4,8,16,32\}$, where $K=32$ is treated as a reference "exact" solution, i.e.,

$$
\operatorname{error}_{K, L_{2}(\Omega)}:=\left\|u_{K}-u_{32}\right\|_{L_{2}(\Omega)}, \quad \operatorname{error}_{K, H^{1}(\Omega)}:=\left\|u_{K}-u_{32}\right\|_{H^{1}(\Omega)},
$$

where $u_{K}:=u_{h}$ for the grid parameter $K$.

The nonlinear discrete problem was solved by Newton's method with step scaling relying on the Picard method. More details on the nonlinear solver used in our simulations may be found in [23].

Results of these simulations are presented in Table 9.2. We observe a linear reduction of the $H^{1}$-error, which is consistent with our theoretical result, as the $H^{1}$-norm is bounded by the broken norm up to a constant factor. We also note the quadratic $L_{2}$-norm convergence rate. These results were obtained for the penalty parameter $\sigma_{e}=3 \times 10^{6}$.

As can be observed in Figure 9.2, in this case, the solution has a one-dimensional nature. To study a more sophisticated behavior, we introduce a second device with a more complex structure (Figure 9.3, see Table 9.3 for "material" parameters and grid description). As we see in Table 9.4, the convergence rate is similar as in the previous example. 


\section{ETNA}

Kent State University and

Johann Radon Institute (RICAM)

TABLE 9.3

Parameters of the second device used in the simulations.

\begin{tabular}{lrrrrc} 
Param. & $\Omega_{1}, \Omega_{2}, \Omega_{3}, \Omega_{4}, \Omega_{7}$ & $\Omega_{5}, \Omega_{6}, \Omega_{8}, \Omega_{9}$ & Grid & $N_{x}$ & $N_{y}$ \\
\hline Length & $1 \times 10^{-2}$ & $1 \times 10^{-2}$ & $\Omega_{1}$ & $2 K+1$ & $2 K+1$ \\
Width & $1 \times 10^{-2}$ & $1 \times 10^{-2}$ & $\Omega_{2}$ & $2 K+1$ & $2 K+1$ \\
$\varepsilon$ & $3 \times 10^{-3}$ & $1 \times 10^{-3}$ & $\Omega_{3}$ & $2 K+1$ & $2 K+1$ \\
$\mu_{n}$ & $1 \times 10^{3}$ & $3 \times 10^{3}$ & $\Omega_{4}$ & $2 K+1$ & $2 K+1$ \\
$\mu_{p}$ & $1 \times 10^{2}$ & $3 \times 10^{2}$ & $\Omega_{5}$ & $4 K+1$ & $4 K+1$ \\
$k_{1}$ & $3 \times 10^{2}$ & $-3 \times 10^{2}$ & $\Omega_{6}$ & $2 K+1$ & $4 K+1$ \\
$C_{\text {rad }}$ & $1 \times 10^{-3}$ & $2 \times 10^{-3}$ & $\Omega_{7}$ & $2 K+1$ & $2 K+1$ \\
& & & $\Omega_{8}$ & $4 K+1$ & $2 K+1$ \\
& & & $\Omega_{9}$ & $2 K+1$ & $2 K+1$
\end{tabular}

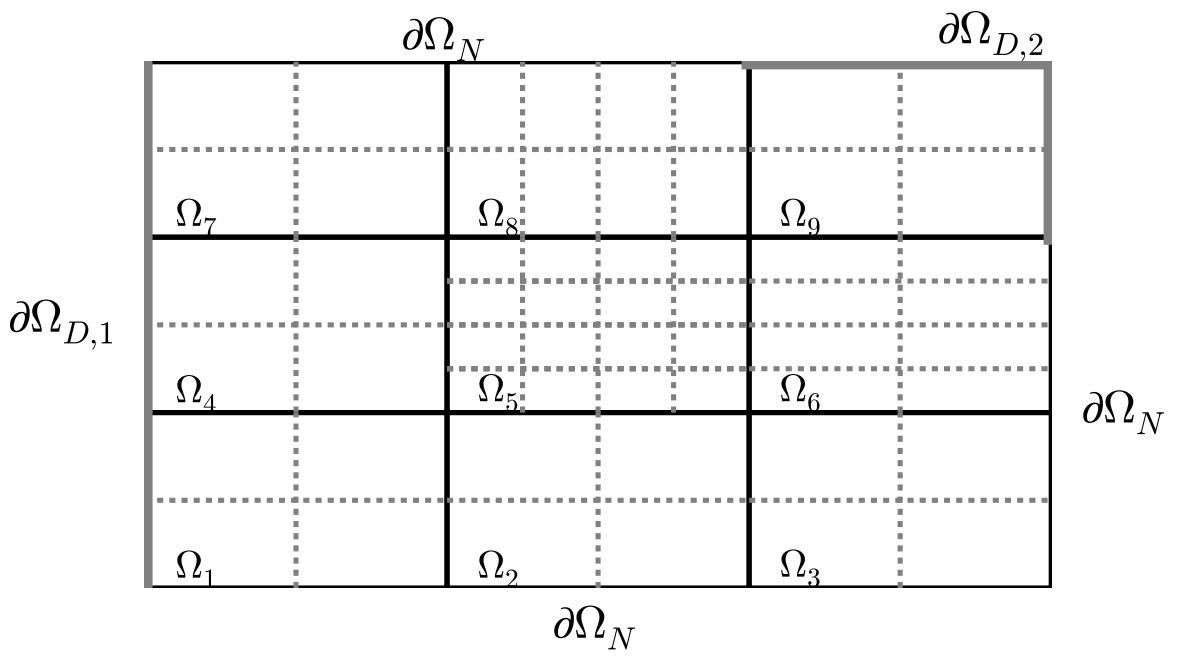

FIG. 9.3. Schema of the second device used in the simulations. The layers $\Omega_{1}, \Omega_{2}, \Omega_{3}, \Omega_{4}, \Omega_{7}$ correspond to the n-type region, while the remainder corresponds to the p-type region. The left contact is attached to the whole left edge, while the right contact is attached to the boundary of $\Omega_{9}$. The grid for $K=1$ is presented with the diagonal lines removed to improve readability.

The theory presented in this paper covers only the equilibrium state described in Section 2. We also performed simulations for the non-equilibrium state. Thus, we use the presented discretization for every equation of the system (1.2). The boundary conditions for the function $u$ are similar as before, i.e., $\left.\hat{u}\right|_{\partial \Omega_{D, 1}}=0$ and $\left.\hat{u}\right|_{\partial \Omega_{D, 2}}=u_{\text {built }}+u_{\text {bias }}$, where $u_{\text {bias }}$ is a nonzero difference potential between the electrodes, called the bias. For the functions $v, w$, we impose two implicit conditions on $\partial \Omega_{D}:\left.v\right|_{\partial \Omega_{D}}=\left.w\right|_{\partial \Omega_{D}}$ and $\left.\rho\right|_{\partial \Omega_{D}}=0$; cf. (9.1). On $\Omega_{N}$ we impose a homogeneous Neumann boundary condition.

Results of this simulation are presented in Table 9.5. For the function $u$, the results are similar to the equilibrium state. For the functions $v, w$, the convergence is much worse. We may roughly estimate that the $L_{2}$-error reduces linearly, while the $H^{1}$-error convergence rate is sublinear, but the latter is hard to estimate precisely without the exact solution. In the comparison, we also included the functions $n, p$. The van Roosbroeck equations may be formulated in terms of functions $u, v, w$, but from the physical point of view, there are other logical choices possible [21]. Another choice is $u, n, p$ (see (9.2) for the definition of $n, p$ ), as 


\section{ETNA}

Kent State University and

Johann Radon Institute (RICAM)

DISCONTINUOUS GALERKIN METHOD FOR SEMICONDUCTORS

TABLE 9.4

$L_{2}(\Omega)$ - and $H^{1}(\Omega)$-error of $u$ as a function of the grid density parameter $K$ for the second device in equilibrium state. Numbers in brackets denote the error norm reduction factor.

\begin{tabular}{rllll}
$\mathrm{K}$ & \multicolumn{2}{c}{$L_{2}(\Omega)$} & \multicolumn{2}{c}{$H^{1}(\Omega)$} \\
\hline 1 & $2.0 \times 10^{-2}$ & & $1.9 \times 10^{-1}$ & \\
2 & $5.2 \times 10^{-3}$ & $(3.8)$ & $9.5 \times 10^{-2}$ & $(2.0)$ \\
4 & $1.3 \times 10^{-3}$ & $(3.9)$ & $4.8 \times 10^{-2}$ & $(2.0)$ \\
8 & $3.5 \times 10^{-4}$ & $(3.8)$ & $2.4 \times 10^{-2}$ & $(2.0)$ \\
16 & $9.4 \times 10^{-5}$ & $(3.7)$ & $1.2 \times 10^{-2}$ & $(2.0)$ \\
32 & $2.3 \times 10^{-5}$ & $(4.0)$ & $5.4 \times 10^{-3}$ & $(2.2)$
\end{tabular}

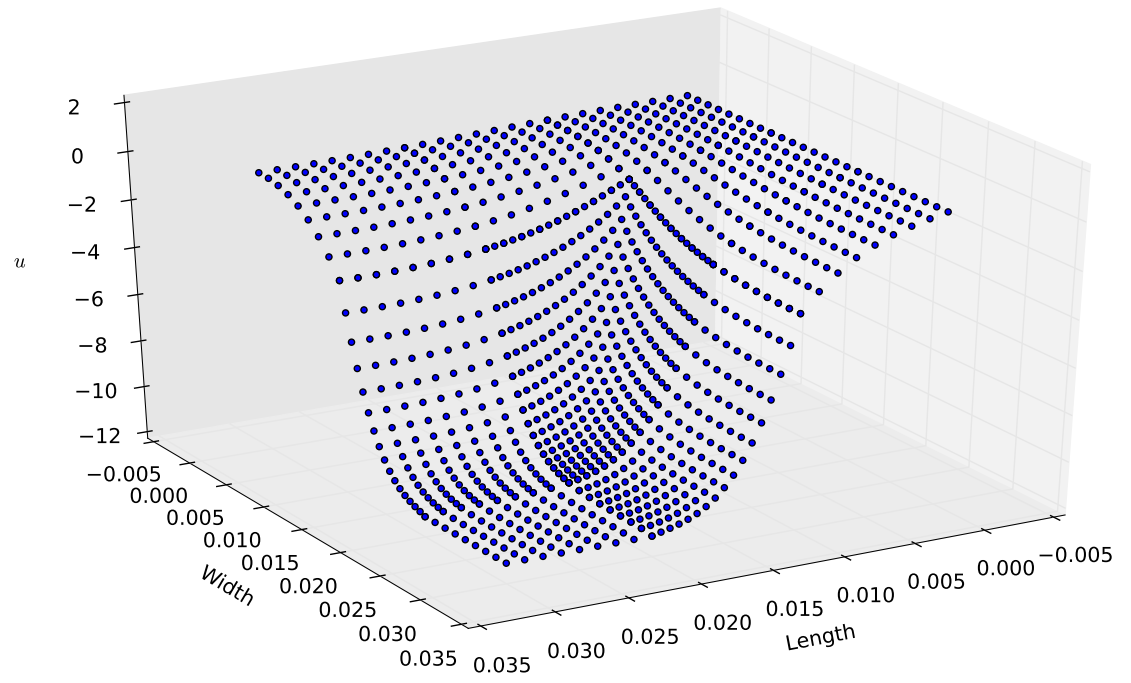

FIG. 9.4. The function $u$ for the second example in the equilibrium state for $K=4$.

the charge $\rho$ and many recombination models (radiative, Shockley-Read-Hall, Auger) can be easily expressed in terms of these functions.

We observe that the error convergence for $n, p$ is faster than for $v, w$, similar to that of the function $u$. Thus, the determination of the physical parameters like the recombination rate, current, or optical power may rely on the better precision of functions $n, p$ despite the slow convergence of functions $v, w$.

10. Conclusions. We have presented a composite discontinuous Galerkin discretization of the drift-diffusion equations, derived from the symmetric interior penalty Galerkin method [22]. The discrete problem is shown to be well-defined, and the error is estimated. In case of a uniform increase of the grid density, the $H^{1}$-norm of the error of the composite symmetric interior penalty Galerkin (CSIPG) method is estimated as $O(h)$. The results of the numerical simulations presented in this paper agree with the theoretical estimates.

Acknowledgements. The authors acknowledge the support of the National Science Centre, Poland by Grant No. DEC-2016/21/B/ST1/00350 and the support of the Polish National Agency for Academic Exchange. 
TABLE 9.5

$L_{2}(\Omega)$ - and $H^{1}(\Omega)$-error of $u, v, w, n, p$ as a function of the grid density parameter $K$ for the second device for $u_{\text {bias }}=8$. The numbers in brackets denote the error norm reduction factor. The functions $n, p$ are defined in (9.2).

\begin{tabular}{rcccc}
$\mathrm{K}$ & \multicolumn{2}{c}{$L_{2}(\Omega)$} & $H^{1}(\Omega)$ \\
\hline \multicolumn{3}{c}{ Function u } \\
1 & $3.1 \times 10^{-2}$ & $3.1 \times 10^{-1}$ \\
2 & $8.6 \times 10^{-3}$ & $(3.6)$ & $1.6 \times 10^{-1}$ & $(1.9)$ \\
4 & $2.6 \times 10^{-3}$ & $(3.3)$ & $8.0 \times 10^{-2}$ & $(2.0)$ \\
8 & $9.8 \times 10^{-4}$ & $(2.7)$ & $4.0 \times 10^{-2}$ & $(2.0)$ \\
16 & $4.0 \times 10^{-4}$ & $(2.5)$ & $2.0 \times 10^{-2}$ & $(2.0)$ \\
32 & $1.3 \times 10^{-4}$ & $(3.1)$ & $8.8 \times 10^{-3}$ & $(2.2)$ \\
\hline
\end{tabular}

Function $v$

\begin{tabular}{rrrrr}
1 & $1.7 \times 10^{-1}$ & & $9.8 \times 10^{-1}$ & \\
2 & $9.7 \times 10^{-2}$ & $(1.7)$ & $9.6 \times 10^{-1}$ & $(1.0)$ \\
4 & $5.7 \times 10^{-2}$ & $(1.7)$ & $9.1 \times 10^{-1}$ & $(1.0)$ \\
8 & $3.2 \times 10^{-2}$ & $(1.8)$ & $8.5 \times 10^{-1}$ & $(1.1)$ \\
16 & $1.7 \times 10^{-2}$ & $(1.9)$ & $7.5 \times 10^{-1}$ & $(1.1)$ \\
32 & $6.9 \times 10^{-3}$ & $(2.4)$ & $5.7 \times 10^{-1}$ & $(1.3)$ \\
\hline
\end{tabular}

Function $w$

\begin{tabular}{rrrrr}
1 & $5.8 \times 10^{-1}$ & & $9.7 \times 10^{-1}$ & \\
2 & $3.5 \times 10^{-1}$ & $(1.6)$ & $9.5 \times 10^{-1}$ & $(1.0)$ \\
4 & $2.1 \times 10^{-1}$ & $(1.6)$ & $9.1 \times 10^{-1}$ & $(1.0)$ \\
8 & $1.3 \times 10^{-1}$ & $(1.7)$ & $8.5 \times 10^{-1}$ & $(1.1)$ \\
16 & $6.8 \times 10^{-2}$ & $(1.9)$ & $7.6 \times 10^{-1}$ & $(1.1)$ \\
32 & $2.8 \times 10^{-2}$ & $(2.4)$ & $5.9 \times 10^{-1}$ & $(1.3)$ \\
\hline
\end{tabular}

Function $n$

\begin{tabular}{rrrrr}
1 & $4.7 \times 10^{-2}$ & & $4.5 \times 10^{-1}$ & \\
2 & $1.5 \times 10^{-2}$ & $(3.1)$ & $2.7 \times 10^{-1}$ & $(1.7)$ \\
4 & $5.0 \times 10^{-3}$ & $(3.0)$ & $1.5 \times 10^{-1}$ & $(1.8)$ \\
8 & $1.7 \times 10^{-3}$ & $(2.9)$ & $7.6 \times 10^{-2}$ & $(2.0)$ \\
16 & $6.0 \times 10^{-4}$ & $(2.9)$ & $3.8 \times 10^{-2}$ & $(2.0)$ \\
32 & $1.7 \times 10^{-4}$ & $(3.5)$ & $1.7 \times 10^{-2}$ & $(2.2)$ \\
\hline
\end{tabular}

Function $p$

$\begin{array}{rrrrr}1 & 3.3 \times 10^{-2} & & 2.5 \times 10^{-1} & \\ 2 & 1.1 \times 10^{-2} & (3.0) & 1.2 \times 10^{-1} & (2.1) \\ 4 & 4.6 \times 10^{-3} & (2.4) & 5.9 \times 10^{-2} & (2.0) \\ 8 & 1.9 \times 10^{-3} & (2.4) & 3.0 \times 10^{-2} & (2.0) \\ 16 & 7.1 \times 10^{-4} & (2.7) & 1.5 \times 10^{-2} & (2.0) \\ 32 & 2.0 \times 10^{-4} & (3.5) & 6.5 \times 10^{-3} & (2.3)\end{array}$




\section{ETNA}

Kent State University and

Johann Radon Institute (RICAM)

\section{REFERENCES}

[1] R. E. BANK AND D. J. Rose, Some error estimates for the box method, SIAM J. Numer. Anal., 24 (1987), pp. 777-787.

[2] S. C. BRENNER, Poincare-Friedrichs inequalities for piecewise H1 functions, SIAM J. Numer. Anal., 41 (2003), pp. 306-324.

[3] S. C. BREnNER And L. R. ScotT, The Mathematical Theory of Finite Element Methods, 3rd. ed., Springer, New York, 2008.

[4] R. K. COOMER AND I. G. GRAHAM, Massively parallel methods for semiconductor device modelling, Computing, 56 (1996), pp. 1-27.

[5] D. A. Di Pietro AND A. ERN, Mathematical Aspects of Discontinuous Galerkin Methods, Springer, Heidelberg, 2012.

[6] M. DRYJA, On discontinuous Galerkin methods for elliptic problems with discontinuous coefficients, Comput. Methods Appl. Math., 3 (2003), pp. 76-85.

[7] M. DRYJA, J. GALVIS, AND M. SARKIS, A FETI-DP preconditioner for a composite finite element and discontinuous Galerkin method, SIAM J. Numer. Anal., 51 (2013), pp. 400-422.

[8] S. GIANI, Solving elliptic eigenvalue problems on polygonal meshes using discontinuous Galerkin composite finite element methods, Appl. Math. Comput., 267 (2015), pp. 618-631.

[9] V. GiRAult And P.-A. RAVIART, Finite Element Methods for Navier-Stokes equations, Springer, Berlin, 1986.

[10] V. Girault, B. Rivière, And M. F. WheEler, A discontinuous Galerkin method with nonoverlapping domain decomposition for the Stokes and Navier-Stokes problems, Math. Comp., 74 (2005), pp. 53-84.

[11] H. K. GUMMEL, A self-consistent iterative scheme for one-dimensional steady state transistor calculations, IEEE Trans. Electron. Dev., 11 (1964), pp. 455-464.

[12] J. W. JEROME, Consistency of semiconductor modeling: an existence/stability analysis for the stationary Van Roosbroeck system, SIAM J. Appl. Math., 45 (1985), pp. 565-590.

[13] - Analysis of Charge Transport, Springer, Berlin, 1996.

[14] T. KERKHOVEN AND Y. SAAD, On acceleration methods for coupled nonlinear elliptic systems, Numer. Math., 60 (1992), pp. 525-548.

[15] J.-L. Lions, Quelques Méthodes de Résolution des Problèmes aux Limites Non Linéaires, Dunod/GauthierVillars, Paris, 1969.

[16] A. F. D. Loula, M. R. Correa, J. N. C. Guerreiro, And E. M. Toledo, On finite element methods for heterogeneous elliptic problems, Internat. J. Solids Structures, 45 (2008), pp. 6436-6450.

[17] L. MACHIELS, A posteriori finite element bounds for output functionals of discontinuous Galerkin discretizations of parabolic problems, Comput. Methods Appl. Mech. Engrg., 190 (2001), pp. 3401-3411.

[18] P. A. Markowich, C. A. Ringhofer, And C. Schmeiser, Semiconductor Equations, Springer, Vienna, 1990.

[19] J. J. H. Miller, W. H. A. Schilders, And S. WAng, Application of finite element methods to the simulation of semiconductor devices, Rep. Prog. Phys., 62 (1999), pp. 277-353.

[20] M. S. Mоск, On equations describing steady-state carrier distributions in a semiconductor device, Comm. Pure Appl. Math., 25 (1972), pp. 781-792.

[21] S. J. Polak, C. DEN HeiJer, W. H. A. Schilders, AND P. MARKOWICH, Semiconductor device modelling from the numerical point of view, Internat. J. Numer. Methods Engrg., 24 (1987), pp. 763-838.

[22] B. RiviERE, Discontinuous Galerkin Methods for Solving Elliptic and Parabolic Equations: Theory and Implementation, SIAM, Philadelphia, 2008.

[23] K. Sakowski, L. Marcinkowski, And S. KRUKOWski, Modification of the Newton's method for the simulations of gallium nitride semiconductor devices, in Parallel Processing and Applied Mathematics, Part II, R. Wyrzykowski, J. Dongarra, K. Karczewski, and J. Waśniewski, eds., vol. 8385 of Lecture Notes in Comput. Sci., Springer, Berlin, 2014, pp. 551-560.

[24] K. Sakowski, L. Marcinkowski, S. Krukowski, S. Grzanka, and E. Litwin-Staszewska, Simulation of trap-assisted tunneling effect on characteristics of gallium nitride diodes, J. Appl. Phys., 111 (2012), Art. 123115, 7 pages.

[25] K. Sakowski, L. Marcinkowski, P. Strak, P. Kempisty, and S. Krukowski, Discretization of the drift-diffusion equations with the composite discontinuous Galerkin method, in Parallel Processing and Applied Mathematics. Part II, R. Wyrzykowski, E. Deelman, J. Dongarra, K. Karczewski, J. Kitowski, and K. Wiatr, eds., vol. 9574 of Lecture Notes in Comput. Sci., Springer, Cham, 2016, pp. 391-400.

[26] S. Selberherr, Analysis and Simulation of Semiconductor Devices, Springer, Wien, 1984.

[27] S. SzE AND K. NG, Physics of Semiconductor Devices, Wiley, Hoboken, 2006.

[28] A. Toselli And O. Widlund, Domain Decomposition Methods-Algorithms and Theory, Springer, Berlin, 2005.

[29] W. VAN RoOSBROECK, Theory of flow of electrons and holes in germanium and other semiconductors, Bell System Tech. J., 29 (1950), pp. 560-607. 
ETNA

Kent State University and

Johann Radon Institute (RICAM)

\section{K. SAKOWSKI ET AL.}

[30] P. Wilkes, Solid State Theory in Metallurgy, Cambridge University Press, Cambridge, 1973. 